\title{
Experimental supplement
}

General procedure for pyrylium salt synthesis<smiles></smiles><smiles>CC(=O)OC(C)=O</smiles>

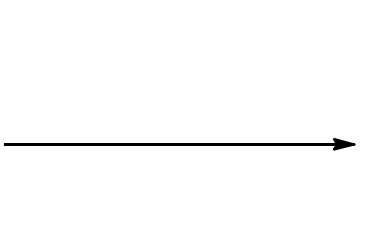<smiles>[R][R]1cccc(-c2cc(C)[o+]c(-c3cc[R1]cc3)c2)c1</smiles>

To substituted acetophenone and acetic anhydride was added boron trifluoride etherate (32.0 $\mathrm{mmol}$ ) at room temperature. The reaction was heated to 135 degrees $\mathrm{C}$ for $4 \mathrm{~h}$, cooled and poured into EtOAc and allowed to stand for $1 \mathrm{~h}$. The yellow solid was filtered and washed with excess EtOAc to give the title compounds as the boron tetrafluoride salt.

General procedure for the condensation reaction with aldehydes<smiles>[R][X]1cccc(-c2cc(C)[o+]c(-c3cc[R1]cc3)c2)c1</smiles><smiles>[R]C=O</smiles><smiles>[R]C=Cc1cc(-c2[R]cccc2)cc(-c2cc[R1]([H])cc2)[o+]1</smiles>

Pyrylium salt $(0.28 \mathrm{mmol})$ and aldehyde $(0.34 \mathrm{mmol})$ in $\mathrm{MeOH}(8 \mathrm{~mL})$ was heated to reflux for $4 \mathrm{~h}$. The reaction was cooled, reduced in vacuo, poured into EtOAc and allowed to stand for $1 \mathrm{~h}$. The dark solid was filtered and washed with excess EtOAc to give the title compounds as the boron tetrafluoride salt.

2-methyl-4,6-diphenylpyrylium boron tetrafluoride salt $\mathbf{6 j c 2 6}$<smiles>CC(=O)c1ccccc1</smiles><smiles>CC(=O)OC(=O)O</smiles><smiles>[B-]CC</smiles><smiles>Cc1cc(-c2ccccc2)cc(-c2ccccc2)[o+]1</smiles> 
To acetophenone (2 g, $16.67 \mathrm{mmol})$ and acetic anhydride $(1.57 \mathrm{~mL}, 16.67 \mathrm{mmol})$ was added boron trifluoride etherate $(3.40 \mathrm{~mL}, 22.50 \mathrm{mmol})$ at room temperature. The reaction was heated to 135 degrees $\mathrm{C}$ for $2 \mathrm{hr}$, cooled, poured into EtOAc and the yellow solid filtered. Recrystallization from AcOH gave yellow solid $813 \mathrm{mg}$.

$\delta_{\mathrm{H}}\left(\mathrm{MeOH}-\mathrm{d}_{4}, 400 \mathrm{MHz}\right) 8.95$ (s, $\left.1 \mathrm{H}, \mathrm{Ar}\right), 8.43$ (s, $1 \mathrm{H}, \mathrm{Ar}$ ), 8.41 (d, $2 \mathrm{H}, \mathrm{J}=8.0$ ), 8.30 (d, $2 \mathrm{H}, \mathrm{J}$ = 8.0), $7.82(\mathrm{t}, 2 \mathrm{H}, \mathrm{J}=7.2, \mathrm{Ar}), 7.73(\mathrm{t}, 4 \mathrm{H}, \mathrm{J}=7.6, \mathrm{Ar}), 3.04(\mathrm{~s}, 3 \mathrm{H}, \mathrm{Me}$, exchanges with deuterium over time)

2,4-bis(4-chlorophenyl)-6-methylpyrylium boron tetrafluoride salt 6jc32-1<smiles>CC(=O)c1ccc(Cl)cc1</smiles><smiles>CCOC(C)=O</smiles><smiles>Cc1cc(-c2ccc(Cl)cc2)cc(-c2ccc(Cl)cc2)[o+]1</smiles>

$6 \mathrm{j}^{\mathrm{C}} 32^{-1} 1$

To p-chloro-acetophenone (2 g, $13.0 \mathrm{mmol})$ and acetic anhydride $(1.22 \mathrm{~mL}, 13.0 \mathrm{mmol})$ was added boron trifluoride etherate ( $4 \mathrm{~mL}, 32.0 \mathrm{mmol}$ ) at room temperature. The reaction was heated to 135 degrees $\mathrm{C}$ for $2 \mathrm{hr}$, cooled, poured into EtOAc and the yellow solid filtered to give $644 \mathrm{mg}$.

$\delta_{\mathrm{H}}\left(\mathrm{MeOH}-\mathrm{d}_{4}, 400 \mathrm{MHz}\right) 8.95$ (s, $\left.1 \mathrm{H}, \mathrm{Ar}\right), 8.44$ (s, $1 \mathrm{H}, \mathrm{Ar}$ ), 8.40 (d, $2 \mathrm{H}, \mathrm{J}=8.8$ ), 8.29 (d, $2 \mathrm{H}, \mathrm{J}$ = 8.8), 7.75 (d, 4 H, J = 7.2, Ar), 3.04 (s, 3 H, Me).

2,4-bis(4-methoxyphenyl)-6-methylpyrylium boron tetrafluoride salt 6jc32-2

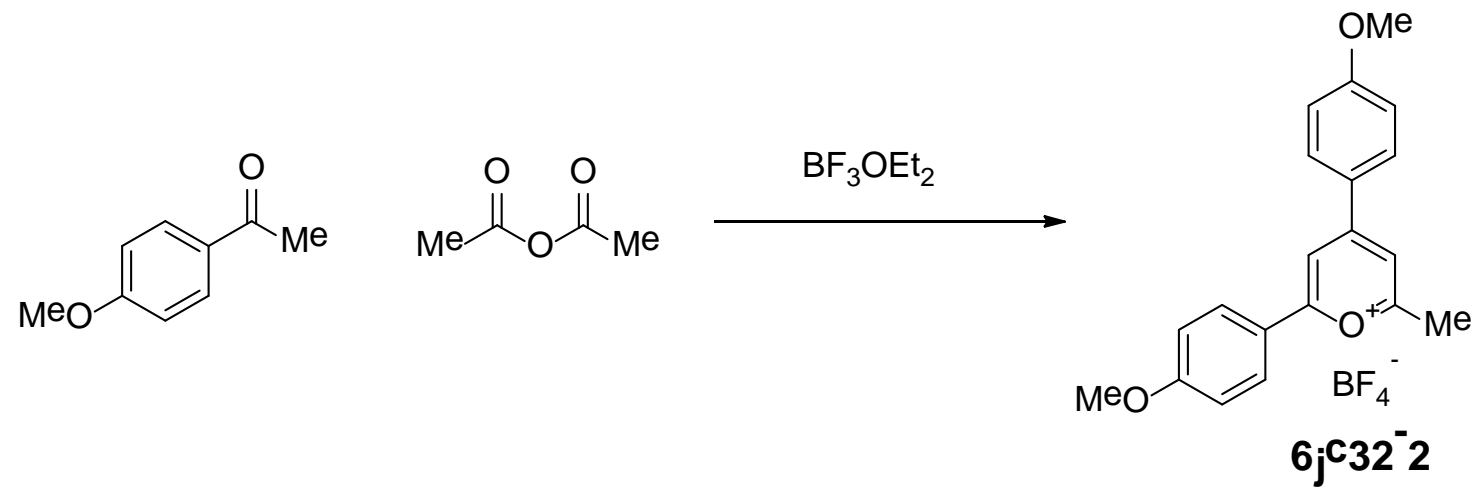


To p-methoxy-acetophenone ( $2 \mathrm{~g}, 13.0 \mathrm{mmol})$ and acetic anhydride $(1.22 \mathrm{~mL}, 13.0 \mathrm{mmol})$ was added boron trifluoride etherate $(4 \mathrm{~mL}, 32.0 \mathrm{mmol})$ at room temperature. The reaction was heated to 135 degrees $\mathrm{C}$ for $2 \mathrm{hr}$, cooled, poured into EtOAc and the red solid filtered to give $313 \mathrm{mg}$.

$\delta_{\mathrm{H}}\left(\mathrm{MeOH}-\mathrm{d}_{4}, 400 \mathrm{MHz}\right) 8.67$ (s, $\left.1 \mathrm{H}, \mathrm{Ar}\right), 8.44$ (s, $1 \mathrm{H}, \mathrm{Ar}$ ), 8.37 (d, $2 \mathrm{H}, \mathrm{J}=9.6$ ), 8.32 (d, $2 \mathrm{H}, \mathrm{J}$ = 9.2), 8.15 (s, $1 \mathrm{H}, \mathrm{Ar}$ ), 7.25-7.21 (m, $4 \mathrm{H}, \mathrm{Ar}$ ), 3.97 (s, 3 H, OMe), 3.96 (s, $3 \mathrm{H}, \mathrm{OMe}$ ), 2.91 (s, $3 \mathrm{H}, \mathrm{Me})$.

2,4,6-trimethylpyrylium boron tetrafluoride salt $6 \mathbf{j c 3 6}$<smiles>CC(C)(O)CC(C)(C)O</smiles>
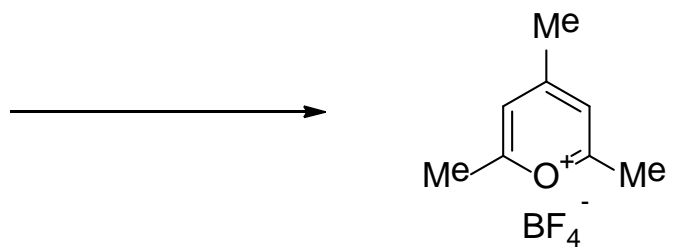

${ }_{6 j} \mathrm{C} 36$

To pentanone (1.16 g, $10.0 \mathrm{mmol})$ and acetic anhydride $(9.5 \mathrm{~mL}, 100.0 \mathrm{mmol})$ was added boron trifluoride etherate $(2.5 \mathrm{~mL}, 10.0 \mathrm{mmol})$ at room temperature. The reaction was heated to 135 degrees $\mathrm{C}$ for $2 \mathrm{hr}$, cooled, poured into EtOAc and the white solid filtered to give $980 \mathrm{mg}$.

$\delta_{\mathrm{H}}\left(\mathrm{MeOH}-d_{4}, 400 \mathrm{MHz}\right) 7.86$ (s, $\left.2 \mathrm{H}, \mathrm{Ar}\right), 2.89$ (s, $6 \mathrm{H}, 2$ x Me), 2.70 (s, $3 \mathrm{H}, \mathrm{Me}$ ).

(E)-4-(4-(dimethylamino)styryl)-2,6-dimethylpyrylium boron tetrafluoride salt 6jc37
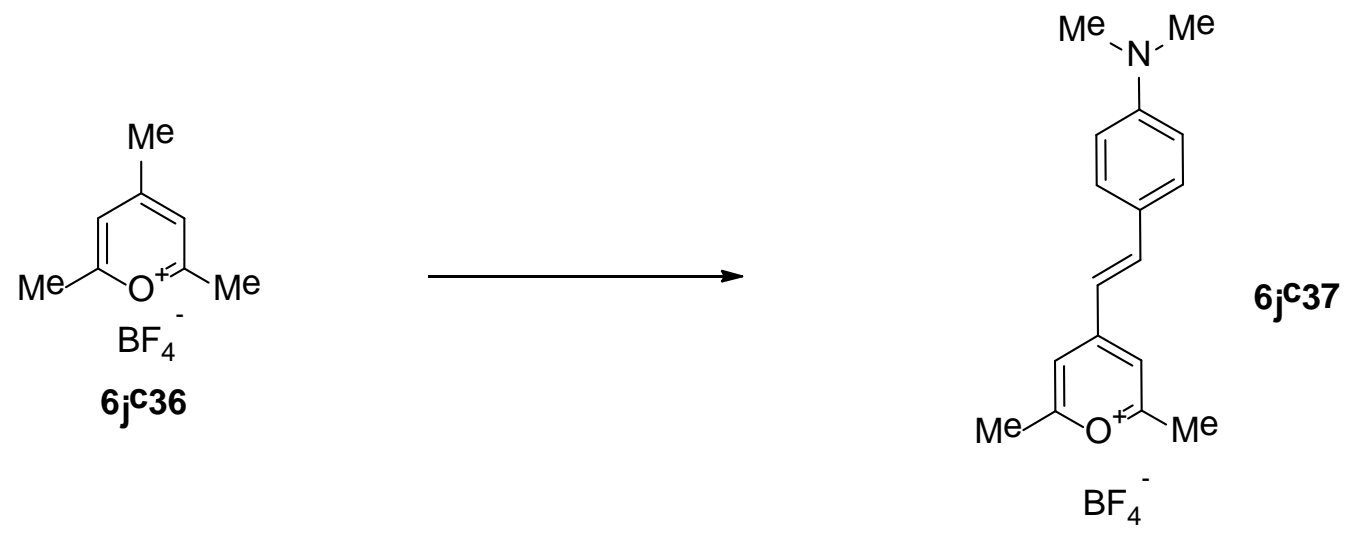

To pyrylium salt 6 jc36 (210 mg, $1.00 \mathrm{mmol}$ ) and p-dimethylamino benzaldehyde (150 mg, 1.00 $\mathrm{mmol})$ in $\mathrm{MeOH}(10 \mathrm{~mL})$ was stirred at room temperature overnight. The solvent was removed and the residue was suspended in ether and filtered to give blue solid. Recrystallization from EtOH gave blue solid of $110 \mathrm{mg}$. 
$\delta_{\mathrm{H}}\left(\mathrm{DMSO}-\mathrm{d}_{6}, 400 \mathrm{MHz}\right) 8.35$ (d, $1 \mathrm{H}, \mathrm{J}=14.8, \mathrm{H}$-vinyl), 7.76 (d, $2 \mathrm{H}, \mathrm{J}=$ 8.4, Ar), 7.69 (s, $2 \mathrm{H}$, Ar), 7.13 (d, 1 H, J = 14.8, H-vinyl), 6.91 (d, 2 H, J = 8.4, Ar), 3.16 (s, 6 H, 2 x Me), 2.63 (s, 6 H, $\left.\mathrm{NMe}_{2}\right)$.

4-((1E,3E)-4-(4-(dimethylamino)phenyl)buta-1,3-dien-1-yl)-2,6-dimethylpyrylium boron tetrafluoride salt $\mathbf{6 j} \mathbf{j} 38$
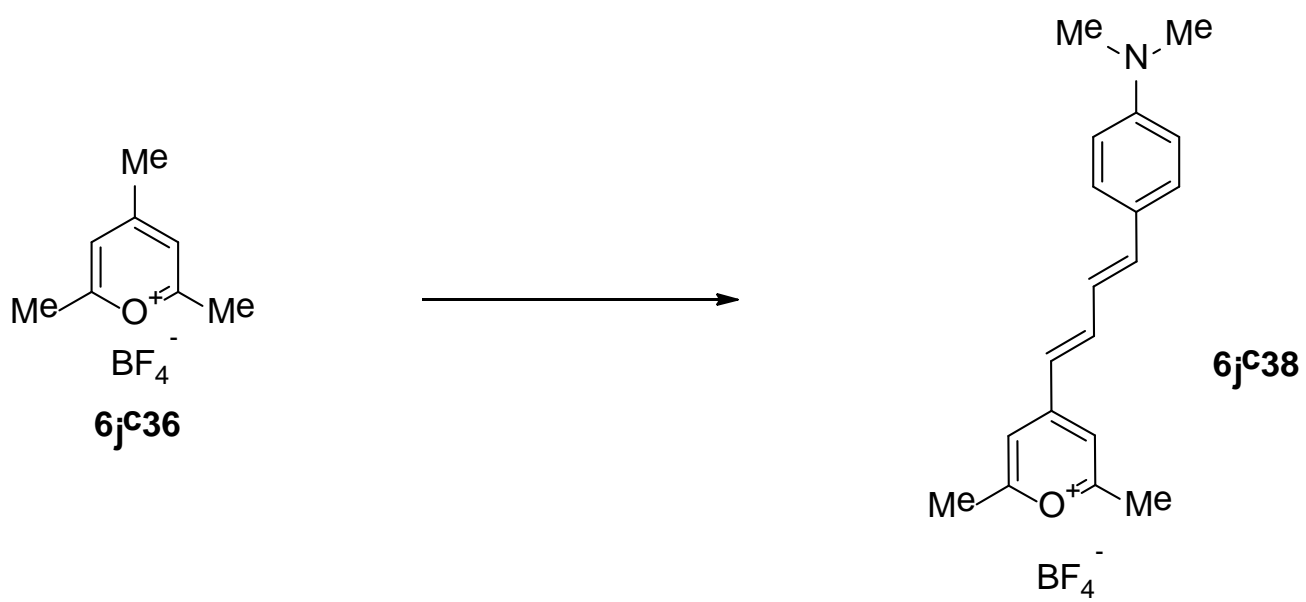

To pyrylium salt 6jc36 (210 mg, $1.00 \mathrm{mmol}$ ) and p-dimethylamino benzaldehyde (176 mg, 1.00 $\mathrm{mmol})$ in $\mathrm{MeOH}(10 \mathrm{~mL})$ was stirred at room temperature overnight. The solvent was removed and the residue was suspended in ether and filtered to give blue solid. Recrystallization from EtOH gave green solid of $110 \mathrm{mg}$.

$\delta_{\mathrm{H}}\left(\mathrm{DMSO}-\mathrm{d}_{6}, 400 \mathrm{MHz}\right) 8.25$ (dd, $1 \mathrm{H}, \mathrm{J}=14.8,14.4$, H-vinyl), 7.73 (s, $2 \mathrm{H}, \mathrm{Ar}$ ), 7.65 (d, 2 H, J = 8.8, Ar), 7.39 (d, $1 \mathrm{H}, \mathrm{J}=14.8$, H-vinyl), 7.27 (t, $1 \mathrm{H}, \mathrm{J}=14.8, \mathrm{H}$-vinyl), 6.81 (d, $2 \mathrm{H}, \mathrm{J}=$ 8.8, Ar), 6.63 (d, 1 H, J = 14.4, H-vinyl), 3.10 (s, 6 H, 2 x Me), 2.64 (s, $6 \mathrm{H}, \mathrm{NMe}_{2}$ ).

(E)-2-(4-(dimethylamino)styryl)-4,6-diphenylpyrylium boron tetrafluoride salt $6 \mathbf{j c 3 9}$<smiles>Cc1cc(-c2ccccc2)cc(-c2ccccc2)[o+]1</smiles><smiles>CN(C)c1ccc(/C=C/c2cc(-c3ccccc3)cc(-c3ccccc3)[o+]2)cc1</smiles> 
Pyrylium salt 6jc26 (100 mg, $0.30 \mathrm{mmol}$ ) and p-dimethylamino benzaldehyde (50 mg, $0.32 \mathrm{mmol}$ ) in $\mathrm{MeOH}(6 \mathrm{~mL})$ was stirred at room temperature overnight. The solvent removed and the residue was suspended in ether and filtered to give green solid of $81 \mathrm{mg}$.

$\delta_{\mathrm{H}}\left(\mathrm{DMSO}-d_{6}, 400 \mathrm{MHz}\right) 8.62$ (s, $1 \mathrm{H}, \mathrm{Ar}$ ), 8.51-8.42 (m, $\left.4 \mathrm{H}, \mathrm{Ar}, \mathrm{HC}=\right), 8.34$ (d, $2 \mathrm{H}, \mathrm{J}=7.2$, Ar), 7.86 (d, 2 H, J = 9.6, Ar), 7.84-7.70 (m, 6 H, Ar), 7.42 (d, 1 H, J = 15.6, HC=), 6.93 (d, 2 H, $\mathrm{J}=8.4$, Ar), 3.17 (s, $6 \mathrm{H}, 2$ x Me).

(E)-2,4-bis(4-chlorophenyl)-6-(4-(dimethylamino)styryl)pyrylium boron tetrafluoride salt 6jc411
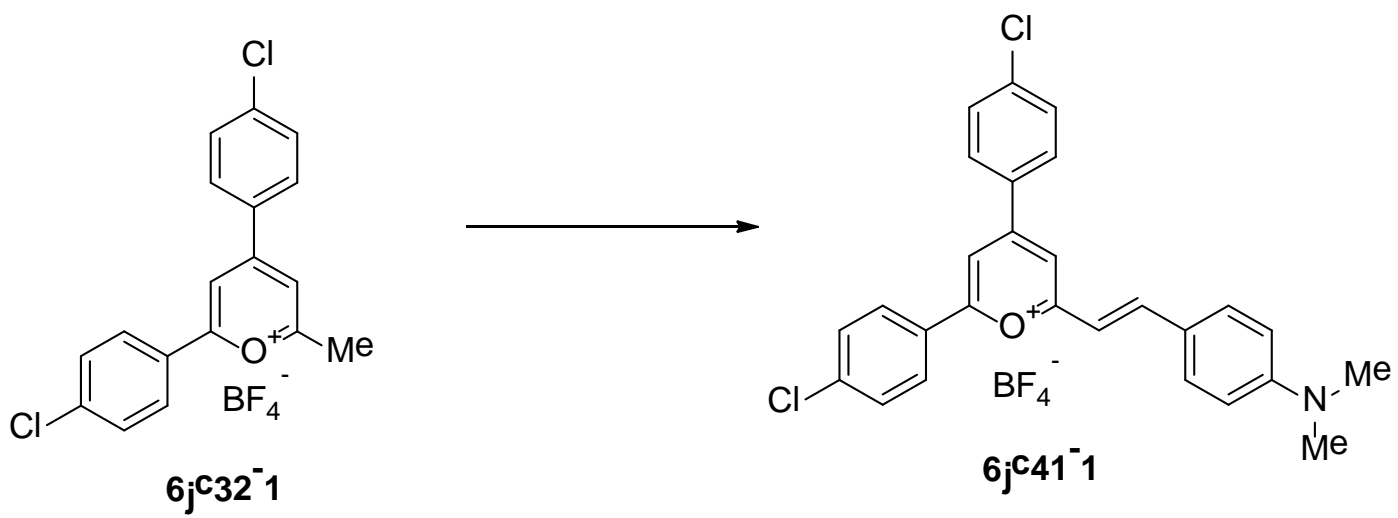

Pyrylium salt 6jc32-1 (134 mg, $0.33 \mathrm{mmol}$ ) and p-dimethylamino benzaldehyde (50 mg, 0.33 $\mathrm{mmol})$ in $\mathrm{MeOH}(6 \mathrm{~mL})$ was stirred at room temperature overnight. The solvent removed and the residue was suspended in ether and filtered to give dark blue solid of $105 \mathrm{mg}$.

$\delta_{\mathrm{H}}\left(\mathrm{DMSO}-d_{6}, 400 \mathrm{MHz}\right) 8.57$ (s, $1 \mathrm{H}, \mathrm{Ar}$ ), 8.49-8.40 (m, $\left.4 \mathrm{H}, \mathrm{Ar}, \mathrm{HC}=\right), 8.34$ (d, $2 \mathrm{H}, \mathrm{J}=8.4$, Ar), 7.84-7.77 (m, 6 H, Ar), 7.37 (d, 1 H, J = 15.6, HC=), 6.92 (d, 2 H, J = 8.4, Ar), 3.17 (s, 6 H, $2 \times \mathrm{Me})$. 
2-((1E,3E)-4-(4-(dimethylamino)phenyl)buta-1,3-dien-1-yl)-4,6-diphenylpyrylium boron tetrafluoride salt $\mathbf{6 j} \mathbf{j} \mathbf{4 3 - 1}$
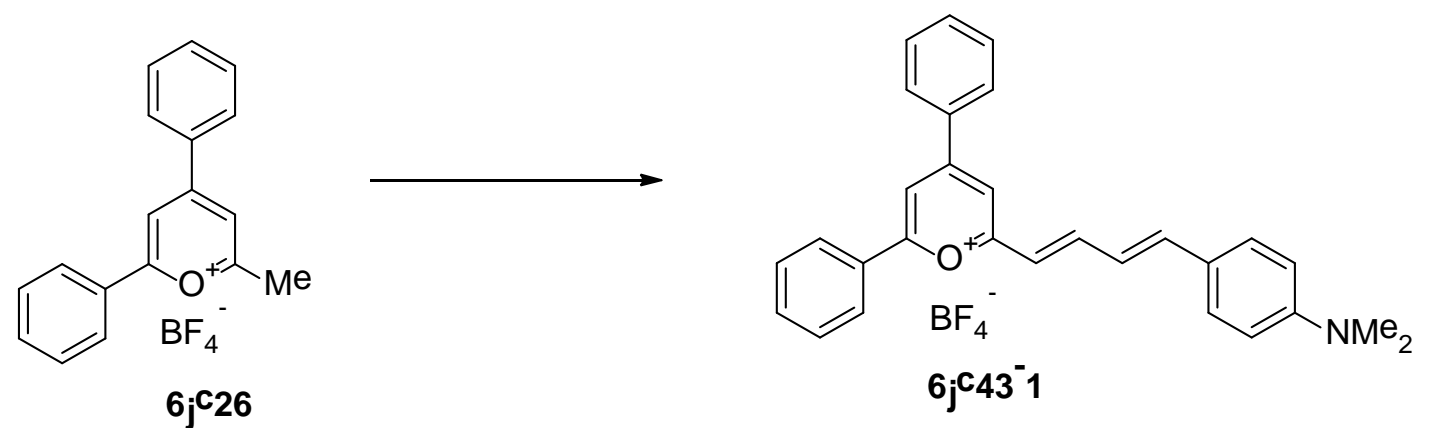

Pyrylium salt 6jc26 (94 mg, $0.28 \mathrm{mmol}$ ) and p-dimethylamino cinnamaldehyde (60 mg, 0.34 $\mathrm{mmol})$ in $\mathrm{MeOH}(8 \mathrm{~mL})$ was heated to reflux for $2 \mathrm{hrs}$. The reaction was cooled, solvent removed and the residue was suspended in ether, washed with EtOAc and filtered to give dark blue solid of $55 \mathrm{mg}$.

$\delta_{\mathrm{H}}\left(\mathrm{DMSO}-d_{6}, 400 \mathrm{MHz}\right) 8.67$ (s, $1 \mathrm{H}, \mathrm{Ar}$ ), 8.54-8.44 (m, $3 \mathrm{H}, \mathrm{Ar}$ ), 8.36 (d, $2 \mathrm{H}, \mathrm{J}=$ 8.0, Ar), 8.31 (t, 1 H, J = 14.8, H-vinyl), 7.85-7.70 (m, 6 H, Ar), 7.65 (d, 2 H, J = 8.4, Ar), 7.57 (d, 1 H, J = 14.8, H-vinyl), 7.31 (t, 1 H, J = 14.8, H-vinyl), 6.88 (d, 1 H, J = 14.8, H-vinyl), 6.83 (d, 2 H, J = 8.4, Ar), 3.09 (s, $6 \mathrm{H}, \mathrm{NMe}_{2}$ ).

2,4-bis(4-chlorophenyl)-6-((1E,3E)-4-(4-(dimethylamino)phenyl)buta-1,3-dien-1-yl)pyrylium boron tetrafluoride salt 6jc43-2

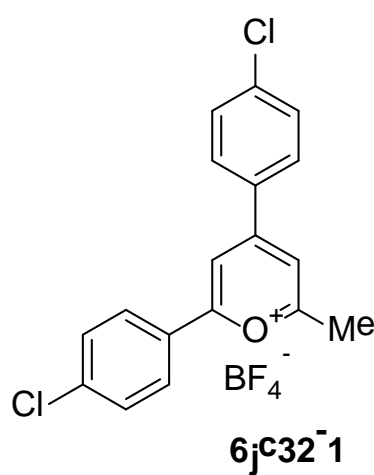
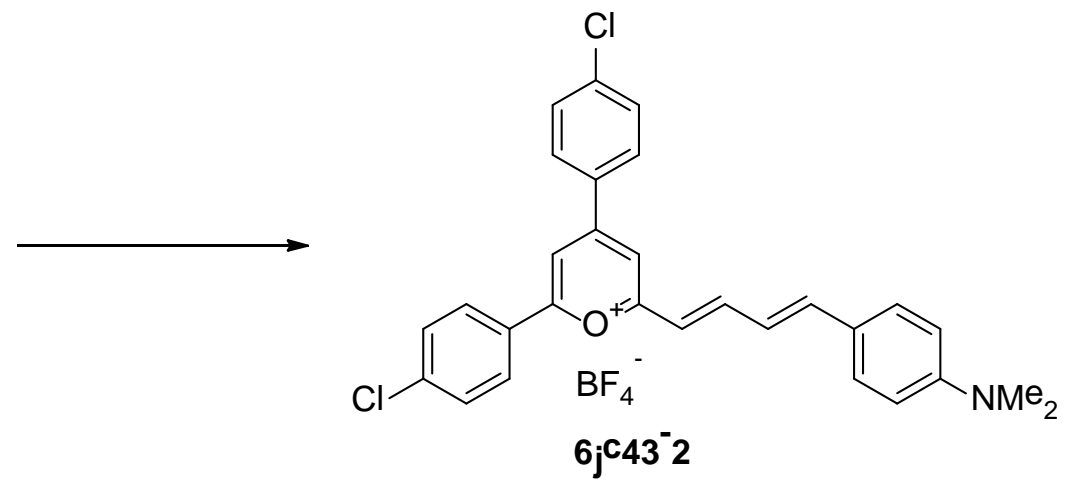

Pyrylium salt 6jc32-1 (112 mg, $0.28 \mathrm{mmol}$ ) and p-dimethylamino cinnamaldehyde (60 mg, 0.34 $\mathrm{mmol})$ in $\mathrm{MeOH}(8 \mathrm{~mL})$ was heated to reflux for $2 \mathrm{hrs}$. The reaction was cooled, solvent removed and the residue was suspended in ether, washed with EtOAc and filtered to give dark blue solid of $46 \mathrm{mg}$. 
$\delta_{\mathrm{H}}\left(\mathrm{DMSO}_{6}, 400 \mathrm{MHz}\right) 8.62$ (s, $1 \mathrm{H}, \mathrm{Ar}$ ), 8.54-8.44 (m, $3 \mathrm{H}, \mathrm{Ar}$ ), 8.37 (d, $2 \mathrm{H}, \mathrm{J}=$ 7.2, Ar), 8.28 (t, 1 H, J = 13.6, H-vinyl), 7.89-7.72 (m, 4 H, Ar), 7.64 (d, 2 H, J = 8.0, Ar), 7.55 (d, 1 H, J = 15.2, H-vinyl), 7.30 (t, 1 H, J = 13.2, H-vinyl), 6.69-6.73 (m, 3 H, Ar, H-vinyl), 3.10 (s, 6 H, $\mathrm{NMe}_{2}$ ).

2,4-bis(4-bromophenyl)-6-methylpyrylium boron tetrafluoride salt 6jc47<smiles>CC(=O)c1ccc(Br)cc1</smiles>

To p-bromo-acetophenone (2.42 g, $12.12 \mathrm{mmol}$ ) and acetic anhydride (1.14 mL, $12.12 \mathrm{mmol}$ ) was added boron trifluoride etherate $(3.68 \mathrm{~mL}, 32.0 \mathrm{mmol})$ at room temperature. The reaction was heated to 135 degrees $\mathrm{C}$ for $2 \mathrm{hr}$, cooled, poured into EtOAc and the yellow solid filtered to give $705 \mathrm{mg}$.

$\delta_{\mathrm{H}}\left(\mathrm{MeOH}-\mathrm{d}_{4}, 400 \mathrm{MHz}\right) 8.95$ (s, $\left.1 \mathrm{H}, \mathrm{Ar}\right), 8.45$ (s, $\left.1 \mathrm{H}, \mathrm{Ar}\right), 8.30$ (d, $2 \mathrm{H}, \mathrm{J}=$ 8.8, Ar), 8.20 (d, 2 $\mathrm{H}, \mathrm{J}=8.8$ ), 8.00-7.86 (m, $4 \mathrm{H}, \mathrm{Ar}$ ), 3.03 (s, $3 \mathrm{H}, \mathrm{Me}$ ).

(E)-2,4-bis(4-bromophenyl)-6-(4-(dimethylamino)styryl)pyrylium boron tetrafluoride salt 6jc481<smiles>Cc1cc(-c2ccc(Br)cc2)cc(-c2ccc(Br)cc2)c1</smiles>

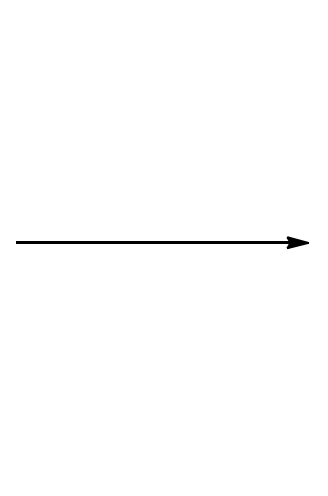<smiles>CN(C)c1ccc(/C=C/c2cc(-c3ccc(Br)cc3)cc(-c3ccc(Br)cc3)[o+]2)cc1</smiles>

To pyrylium salt 6jc47 (100 mg, $0.20 \mathrm{mmol}$ ) and p-dimethylamino benzaldehyde (32 mg, 0.20 $\mathrm{mmol})$ in $\mathrm{MeOH}(10 \mathrm{~mL})$ was stirred at room temperature overnight. The solvent was removed 
and the residue was suspended in ether and filtered to give blue solid. Recrystallization from EtOH gave blue solid of $95 \mathrm{mg}$.

$\delta_{\mathrm{H}}\left(\mathrm{DMSO}-d_{6}, 400 \mathrm{MHz}\right) 8.56(\mathrm{~s}, 1 \mathrm{H}, \mathrm{Ar}), 8.47(\mathrm{~s}, 1 \mathrm{H}, \mathrm{Ar}), 8.43-8.38(\mathrm{~m}, 3 \mathrm{H}, \mathrm{Ar}, \mathrm{HC}=), 8.27$ (d, 2 H, J = 8.4, Ar), 8.00-7.90 (m, 4 H, Ar), 7.84 (d, 2 H, J = 8.4, Ar), 7.39 (d, 1 H, J = 15.6, HC=), 6.94 (d, 2 H, J = 8.8, Ar), 3.18 (s, 6 H, 2 x Me).

2,4-bis(4-bromophenyl)-6-((1E,3E)-4-(4-(dimethylamino)phenyl)buta-1,3-dien-1-yl)pyrylium boron tetrafluoride salt $\mathbf{6 j} \mathbf{c 4 8 - 2}$
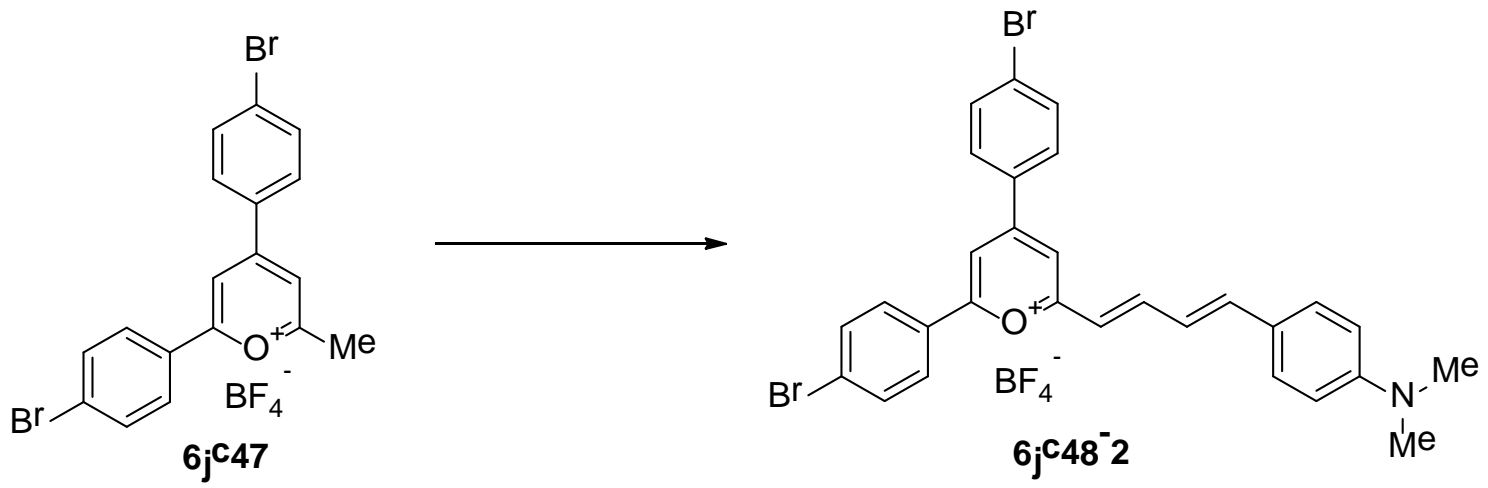

To pyrylium salt 6jc47 (100 mg, $0.20 \mathrm{mmol}$ ) and p-dimethylamino cinnamaldehyde (38 mg, 0.20 $\mathrm{mmol})$ in $\mathrm{MeOH}(10 \mathrm{~mL})$ was stirred at room temperature overnight. The solvent was removed and the residue was suspended in ether and filtered to give blue solid. Recrystallization from EtOH gave blue solid of $41 \mathrm{mg}$.

$\delta_{\mathrm{H}}\left(\mathrm{DMSO}_{6}, d_{6}, 400 \mathrm{MHz}\right) 8.64$ (s, $1 \mathrm{H}, \mathrm{Ar}$ ), 8.49 (s, $1 \mathrm{H}, \mathrm{Ar}$ ), 8.39 (d, $2 \mathrm{H}, \mathrm{J}=$ 8.4, Ar), 8.35-8.24 (m, 3 H, Ar, HC=), 8.00-7.89 (m, 4 H, Ar), 7.65 (d, 2 H, J = 8.4, Ar), 7.56 (d, 1 H, J = 14.8, HC=), 7.32 (t, $1 \mathrm{H}, \mathrm{J}=14.8, \mathrm{HC}=)$, 6.90-6.81 (m, $3 \mathrm{H}, \mathrm{Ar}, \mathrm{HC}=)$, 3.10 (s, $6 \mathrm{H}, \mathrm{NMe}_{2}$ ).

(E)-2-(2-(1H-indol-3-yl)vinyl)-4,6-diphenylpyrylium boron tetrafluoride salt 6jc49-1
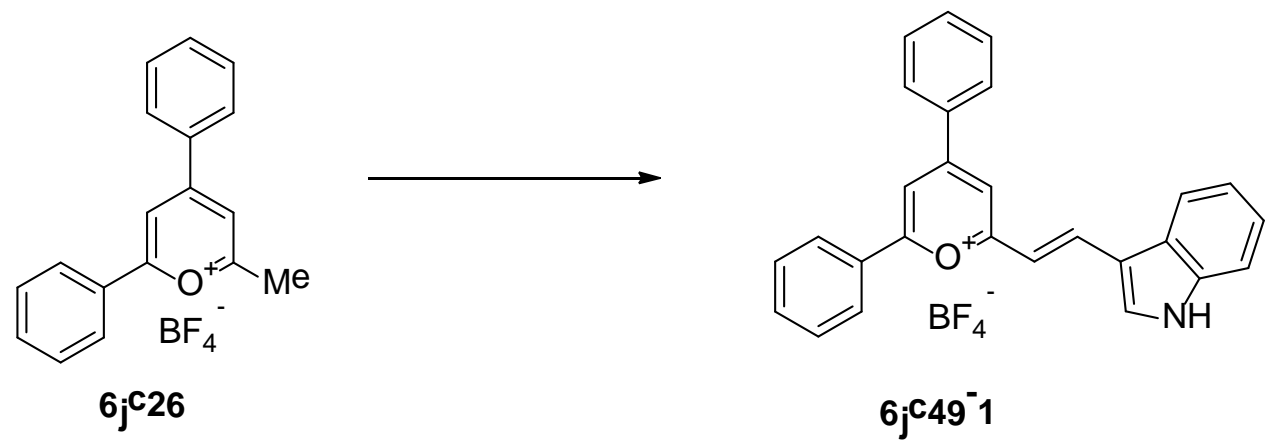
To pyrylium salt 6jc26 (100 mg, $0.30 \mathrm{mmol}$ ) and indole-3-carboxaldehyde (45 mg, $0.30 \mathrm{mmol}$ ) in $\mathrm{MeOH}(8 \mathrm{~mL})$ was stirred at room temperature overnight. The solvent was removed and the residue was suspended in ether and filtered to give blue solid. Recrystallization from EtOH gave blue solid of $32 \mathrm{mg}$.

$\delta_{\mathrm{H}}\left(\mathrm{DMSO}_{6}, 400 \mathrm{MHz}\right) 12.65$ (br s, $\left.1 \mathrm{H}, \mathrm{NH}\right), 8.75$ (d, $\left.1 \mathrm{H}, \mathrm{J}=16.4, \mathrm{HC}=\right), 8.64$ (s, $1 \mathrm{H}, \mathrm{Ar}$ ), 8.60 (s, 1 H, Ar), 8.53 (d, 2 H, J = 7.6, Ar), 8.47 (s, 1 H, Ar), 8.40-8.26 (m, 3 H, Ar), 7.86-7.70 (m, 6 H, Ar), 7.66-7.60 (m, 1 H, Ar), 7.54 (d, 1 H, J = 16.4, HC=), 7.45-7.36 (m, 2 H, Ar).

2-methyl-4,6-di-p-tolylpyrylium boron tetrafluoride salt 6jc50-2<smiles>CC(=O)c1ccc(C)cc1</smiles>

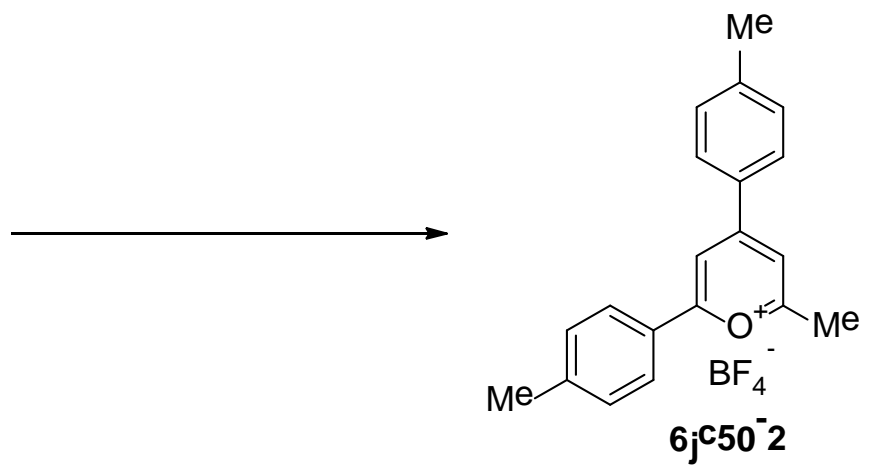

To p-methyl-acetophenone (2.00 g, $15.0 \mathrm{mmol})$ and acetic anhydride $(1.40 \mathrm{~mL}, 15.0 \mathrm{mmol})$ was added boron trifluoride etherate $(4.55 \mathrm{~mL}, 36.9 \mathrm{mmol})$ at room temperature. The reaction was heated to 135 degrees $\mathrm{C}$ for $2 \mathrm{hr}$, cooled, poured into EtOAc and the yellow solid filtered to give $609 \mathrm{mg}$.

$\delta_{\mathrm{H}}\left(\mathrm{MeOH}-\mathrm{d}_{4}, 400 \mathrm{MHz}\right) 8.83$ (s, $\left.1 \mathrm{H}, \mathrm{Ar}\right), 8.32$ (s, $\left.1 \mathrm{H}, \mathrm{Ar}\right), 8.29$ (d, $\left.2 \mathrm{H}, \mathrm{J}=8.0, \mathrm{Ar}\right), 8.20$ (d, 2 $\mathrm{H}, \mathrm{J}=$ 8.0, Ar), 7.59-7.50 (m, 4 H, Ar), 2.98 (s, 3 H, Me), 2.50 (s, 6 H, 2 x Me).

(E)-2-(4-(dimethylamino)styryl)-4,6-di-p-tolylpyrylium boron tetrafluoride salt 6jc51-1
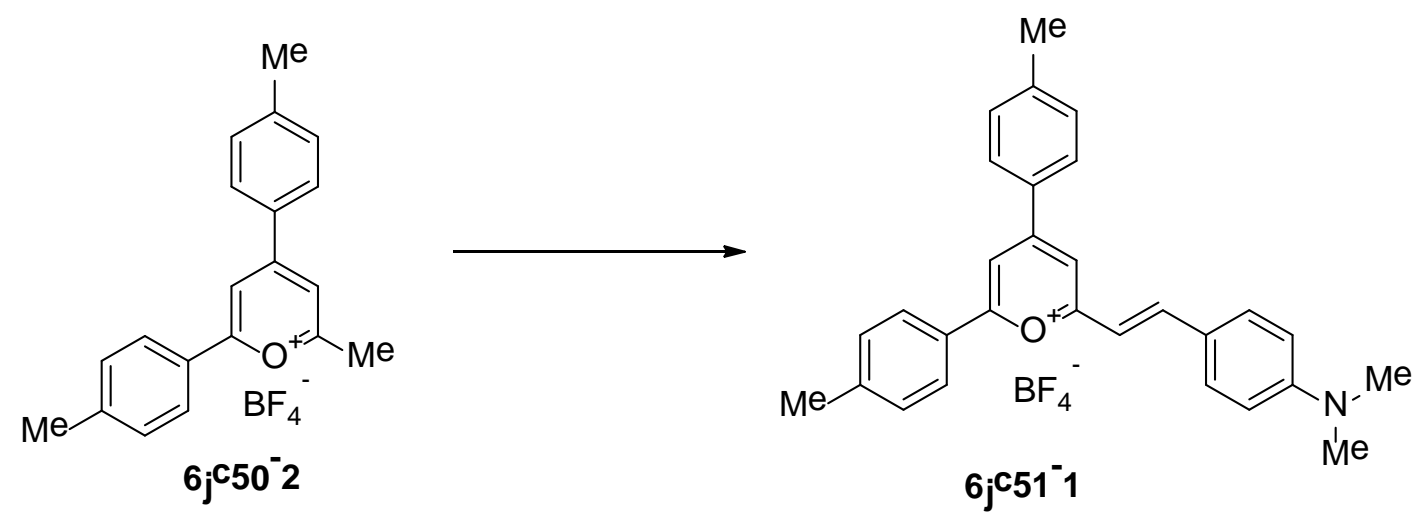
To pyrylium salt 6jc50-2 (100 mg, $0.27 \mathrm{mmol}$ ) and p-dimethylamino benzaldehyde (43 mg, 0.27 $\mathrm{mmol})$ in $\mathrm{MeOH}(8 \mathrm{~mL})$ was stirred at room temperature overnight. The solvent was removed and the residue was suspended in ether and filtered to give blue solid. Recrystallization from EtOH gave blue solid of $73 \mathrm{mg}$.

$\delta_{\mathrm{H}}$ (DMSO-d $\left.d_{6}, 400 \mathrm{MHz}\right) 8.58$ (s, $1 \mathrm{H}, \mathrm{Ar}$ ), 8.43 (s, $1 \mathrm{H}, \mathrm{Ar}$ ), 8.42-8.35 (m, $\left.3 \mathrm{H}, \mathrm{Ar}, \mathrm{HC}=\right), 8.28$ (d, 2 H, J = 8.0, Ar), 7.83 (d, 2 H, J = 8.8, Ar), 7.58-7.50 (m, 4 H, Ar), 7.38 (d, 1 H, J = 15.6, HC=), 6.91 (d, 2 H, J = 8.8, Ar), 3.15 (s, 6 H, NMe $), 2.49$ (s, 6 H, 2 x Me).

2-((1E,3E)-4-(4-(dimethylamino)phenyl)buta-1,3-dien-1-yl)-4,6-di-p-tolylpyrylium boron tetrafluoride salt $\mathbf{6 j} \mathbf{5 1 - 2}$
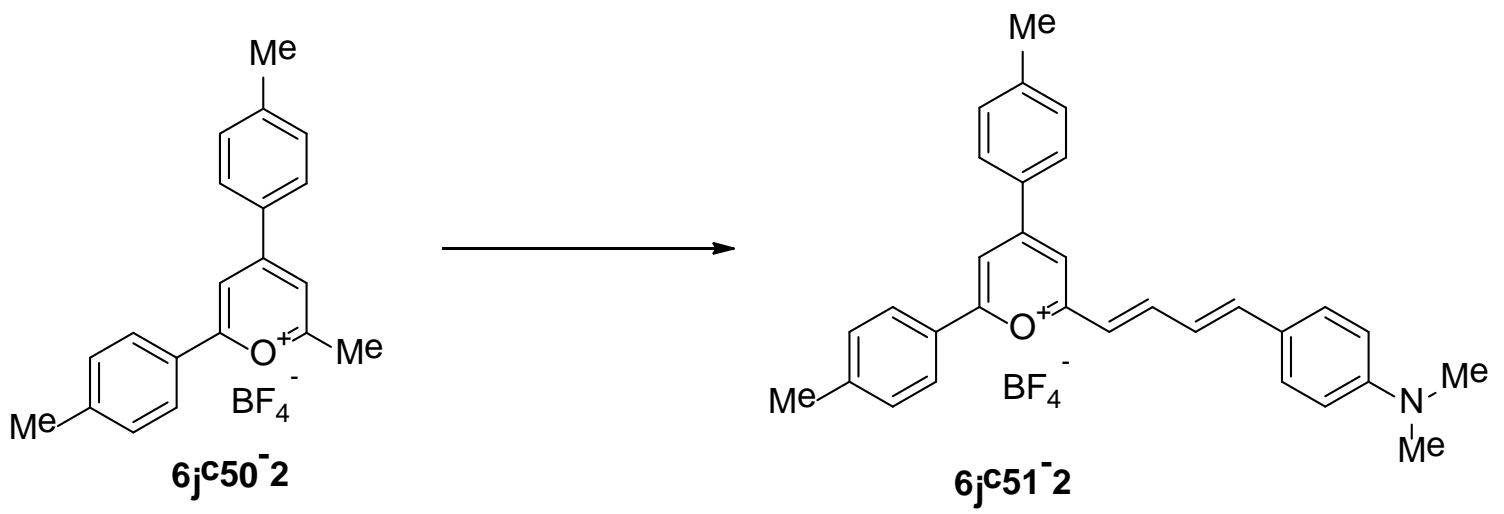

To pyrylium salt 6jc50-2 (100 mg, $0.27 \mathrm{mmol}$ ) and p-dimethylamino cinnamaldehyde (50 mg, $0.27 \mathrm{mmol}$ ) in $\mathrm{MeOH}(8 \mathrm{~mL})$ was stirred at room temperature overnight. The solvent was removed and the residue was suspended in ether and filtered to give blue solid. Recrystallization from EtOH gave blue solid of $58 \mathrm{mg}$.

$\delta_{\mathrm{H}}\left(\mathrm{DMSO}-d_{6}, 400 \mathrm{MHz}\right) 8.63$ (s, $\left.1 \mathrm{H}, \mathrm{Ar}\right), 8.44$ (s, $1 \mathrm{H}, \mathrm{Ar}$ ), 8.39 (d, $2 \mathrm{H}, \mathrm{J}=7.6, \mathrm{Ar}$ ), 8.31 (d, 2 $\mathrm{H}, \mathrm{J}=$ 7.6, Ar), 8.24 (t, $1 \mathrm{H}, \mathrm{HC}=$ ), 7.62 (d, $2 \mathrm{H}, \mathrm{J}=$ 8.8, Ar), 7.58-7.43 (m, $5 \mathrm{H}, \mathrm{Ar}, \mathrm{HC}=), 7.27$ (t, $1 \mathrm{H}, \mathrm{J}=14.8, \mathrm{HC}=$ ), 6.90-6.78 (m, $3 \mathrm{H}, \mathrm{Ar}, \mathrm{HC}=$ ), 3.08 (s, $6 \mathrm{H}, \mathrm{NMe}_{2}$ ), 2.49 (s, $6 \mathrm{H}, 2 \mathrm{x} \mathrm{Me}$ ). 
(E)-2-(2-(1H-indol-3-yl)vinyl)-4,6-bis(4-chlorophenyl)pyrylium boron tetrafluoride salt 6jc53-1
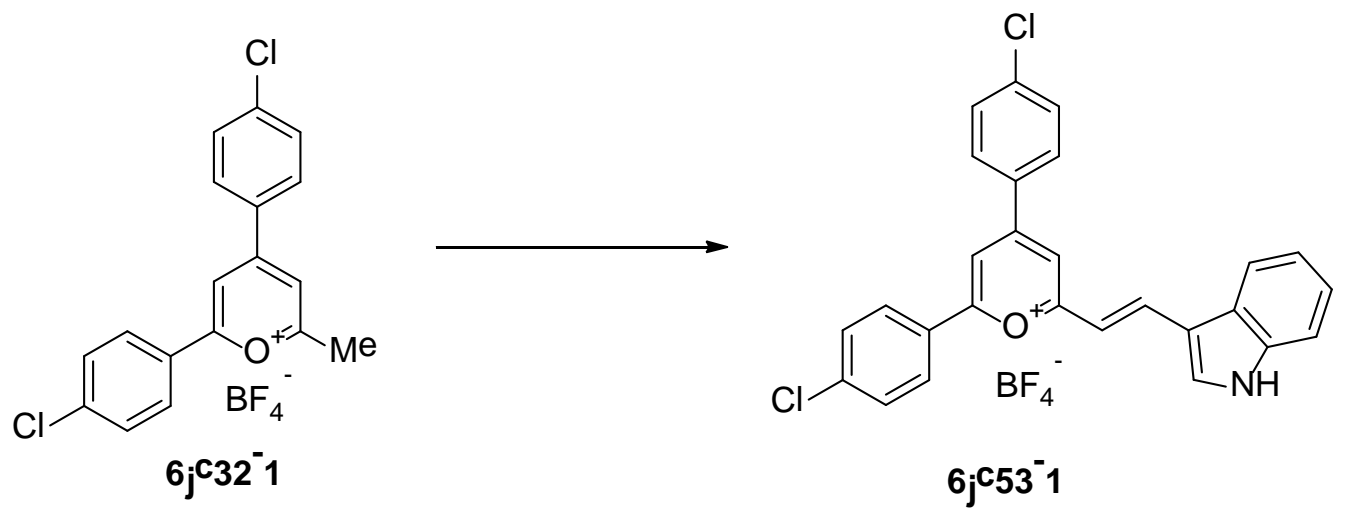

To pyrylium salt 6jc32-1 (138 mg, $0.34 \mathrm{mmol}$ ) and indole-3-carboxaldehyde (50 mg, $0.34 \mathrm{mmol}$ ) in $\mathrm{MeOH}(8 \mathrm{~mL})$ was stirred at room temperature overnight. The solvent was removed and the residue was suspended in ether and filtered to give blue solid. Recrystallization from EtOH gave purple solid of $53 \mathrm{mg}$.

$\delta_{\mathrm{H}}\left(\mathrm{DMSO}-d_{6}, 400 \mathrm{MHz}\right) 12.70$ (br s, $1 \mathrm{H}, \mathrm{NH}$ ), 8.78 (d, $1 \mathrm{H}, \mathrm{J}=14.8, \mathrm{HC}=$ ), 8.64 (s, $1 \mathrm{H}, \mathrm{Ar}$ ), 8.61 (s, 1 H, Ar), 8.53 (d, 2 H, J = 8.4, Ar), 8.47 (s, 1 H, Ar), 8.37 (d, 2 H, J = 8.8, Ar), 8.31-8.26 (m, 1 H, Ar), 7.89-7.80 (m, 4 H, Ar), 7.68-7.60 (m, 1 H, Ar), 7.52 (d, 1 H, J = 14.8, HC=), 7.457.36 (m, $2 \mathrm{H}, \mathrm{Ar})$.

(E)-2-(2-(1H-indol-3-yl)vinyl)-4,6-di-p-tolylpyrylium boron tetrafluoride salt 6jc53-2
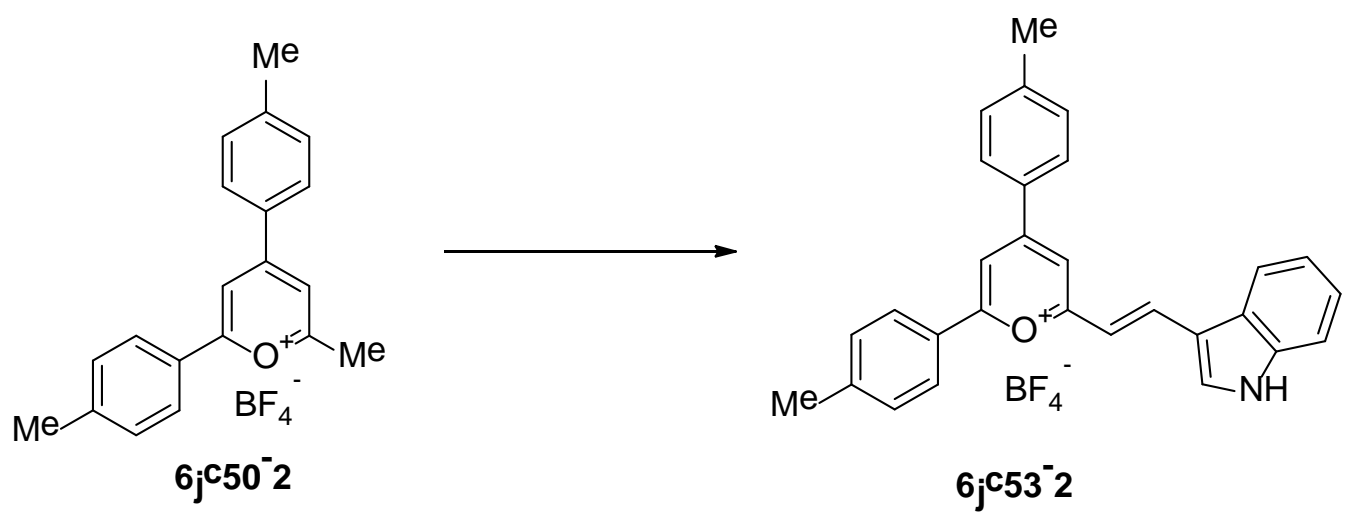

To pyrylium salt 6jc50-2 (123 mg, $0.34 \mathrm{mmol}$ ) and indole-3-carboxaldehyde (50 mg, $0.34 \mathrm{mmol}$ ) in $\mathrm{MeOH}(8 \mathrm{~mL})$ was stirred at room temperature overnight. The solvent was removed and the residue was suspended in ether and filtered to give blue solid. Recrystallization from EtOH gave purple solid of $50 \mathrm{mg}$. 
$\delta_{\mathrm{H}}\left(\mathrm{DMSO}-d_{6}, 400 \mathrm{MHz}\right) 12.55$ (br s, $\left.1 \mathrm{H}, \mathrm{NH}\right), 8.68$ (d, $\left.1 \mathrm{H}, \mathrm{J}=15.6 \mathrm{HC}=\right), 8.60$ (s, $1 \mathrm{H}, \mathrm{Ar}$ ), 8.55 (s, 1 H, Ar), 8.46-8.40 (m, 3 H, Ar), 8.30-8.22 (m, 3 H, Ar), 7.66-7.53 (m, 5 H, Ar), 7.50 (d, $1 \mathrm{H}, \mathrm{J}=15.6, \mathrm{HC}=)$, 7.43-7.35 (m, $2 \mathrm{H}, \mathrm{Ar}), 2.50$ (2 x Me).

(E)-2-(2-(1H-indol-3-yl)vinyl)-4,6-bis(4-bromophenyl)pyrylium boron tetrafluoride salt 6jc53-3<smiles>Cc1cc(-c2ccc(Br)cc2)cc(-c2ccc(Br)cc2)[o+]1</smiles>

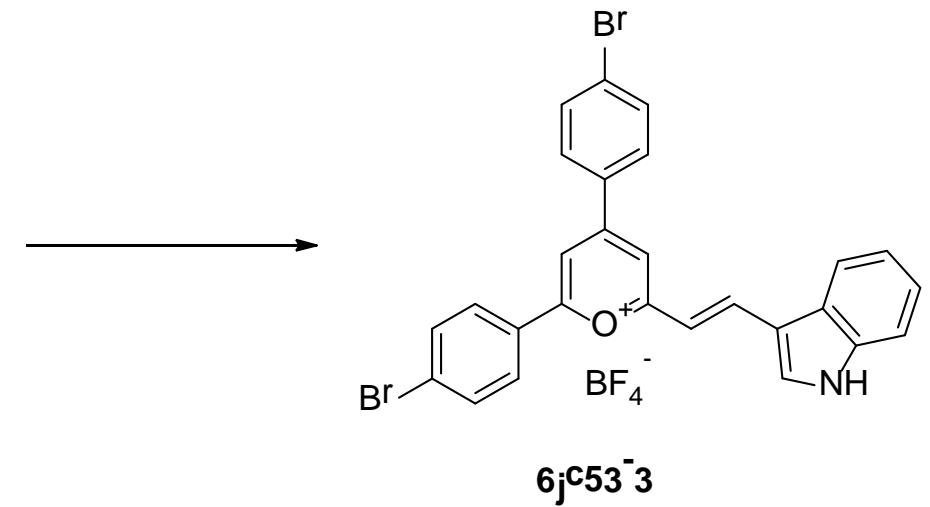

To pyrylium salt 6jc47 (169 mg, $0.34 \mathrm{mmol}$ ) and indole-3-carboxaldehyde (50 mg, $0.34 \mathrm{mmol}$ ) in $\mathrm{MeOH}(8 \mathrm{~mL})$ was stirred at room temperature overnight. The solvent was removed and the residue was suspended in ether and filtered to give blue solid. Recrystallization from EtOH gave purple solid of $38 \mathrm{mg}$.

$\delta_{\mathrm{H}}\left(\mathrm{DMSO}-d_{6}, 400 \mathrm{MHz}\right) 12.70$ (br s, $\left.1 \mathrm{H}, \mathrm{NH}\right), 8.79$ (d, $\left.1 \mathrm{H}, \mathrm{J}=14.8, \mathrm{HC}=\right), 8.64$ (s, $1 \mathrm{H}, \mathrm{Ar}$ ), 8.62 (s, 1 H, Ar), 8.50-8.42 (m, 3 H, Ar), 8.43-8.24 (m, 3 H, Ar), 8.04-7.92 (m, 4 H, Ar), 7.66-7.60 (m, $1 \mathrm{H}, \mathrm{Ar}), 7.52$ (d, $1 \mathrm{H}, \mathrm{J}=14.8, \mathrm{HC}=)$, 7.45-7.36 (m, $2 \mathrm{H}, \mathrm{Ar})$.

2,4-bis(4-(tert-butyl)phenyl)-6-methylpyrylium boron tetrafluoride salt $\mathbf{6 j c 5 6}$<smiles>CC(=O)c1ccc(C(C)(C)C)cc1</smiles><smiles>Cc1cc(-c2ccc(C(C)(C)C)cc2)cc(-c2ccc(C(C)(C)C)cc2)[o+]1</smiles> 
To p-tButyl-acetophenone (2.00 g, $11.4 \mathrm{mmol}$ ) and acetic anhydride (1.07 mL, $11.4 \mathrm{mmol}$ ) was added boron trifluoride etherate $(3.46 \mathrm{~mL}, 28.0 \mathrm{mmol})$ at room temperature. The reaction was heated to 135 degrees $\mathrm{C}$ for $2 \mathrm{hr}$, cooled, poured into EtOAc and the yellow solid filtered to give $494 \mathrm{mg}$.

$\delta_{\mathrm{H}}\left(\mathrm{MeOH}_{-} d_{4}, 400 \mathrm{MHz}\right) 8.86$ (s, $\left.1 \mathrm{H}, \mathrm{Ar}\right), 8.36-8.32$ (m, $\left.3 \mathrm{H}, \mathrm{Ar}\right), 8.26$ (d, $\left.2 \mathrm{H}, \mathrm{J}=8.8, \mathrm{Ar}\right), 7.77$ (d, 4 H, J = 7.6, Ar), 3.00 (s, $3 \mathrm{H}, \mathrm{Me}$ ), 1.40 (s, $18 \mathrm{H}, 2 \mathrm{x} \mathrm{tBu}$ ).

2,4-bis(3,4-dimethylphenyl)-6-methylpyrylium boron tetrafluoride salt 6jc57<smiles>CC(=O)c1ccc(C)c(C)c1</smiles>

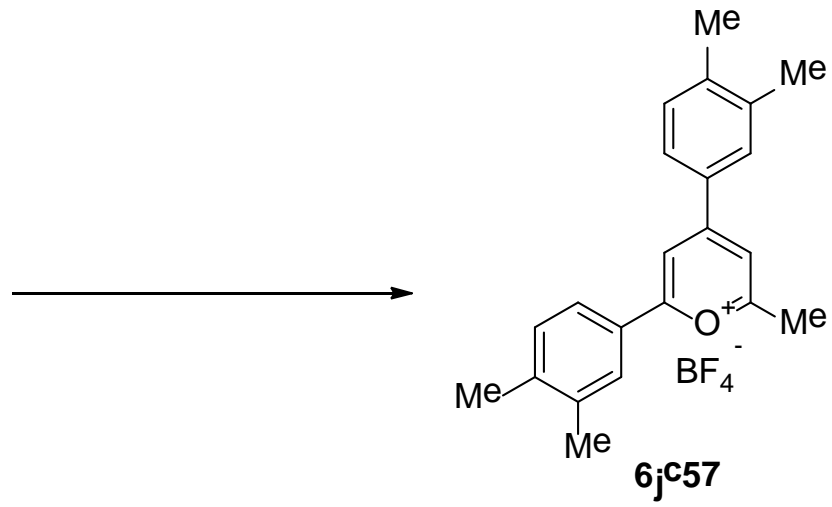

To 3,4-dimethyl-acetophenone (2.00 g, $13.5 \mathrm{mmol})$ and acetic anhydride (1.28 mL, $13.5 \mathrm{mmol})$ was added boron trifluoride etherate $(4.10 \mathrm{~mL}, 33.2 \mathrm{mmol})$ at room temperature. The reaction was heated to 135 degrees $\mathrm{C}$ for $3 \mathrm{hr}$, cooled, poured into EtOAc and the yellow solid filtered to give $417 \mathrm{mg}$.

$\delta_{\mathrm{H}}\left(\mathrm{MeOH}-d_{4}, 400 \mathrm{MHz}\right) 8.82$ (s, $\left.1 \mathrm{H}, \mathrm{Ar}\right), 8.30$ (s, $\left.1 \mathrm{H}, \mathrm{Ar}\right), 8.16-8.09$ (m, $\left.2 \mathrm{H}, \mathrm{Ar}\right), 8.05$ (d, $1 \mathrm{H}$, $\mathrm{J}=$ 7.6, Ar), 7.48 (d, 2 H, J = 8.0, Ar), 2.97 (s, 3 H, Me), 2.44 (s, 6 H, 2 x Me), 2.42 (s, 6 H, 2 x $\mathrm{Me})$.

(E)-2-(2-(1,2,3,5,6,7-hexahydropyrido[3,2,1-ij]quinolin-9-yl)vinyl)-4,6-diphenylpyrylium boron tetrafluoride salt $\mathbf{6 j} \mathbf{5} 58$<smiles>Cc1cc(-c2ccccc2)cc(-c2ccccc2)[o+]1</smiles><smiles>O=Cc1cc2c3c(c1)CCCN3CCC2</smiles><smiles>[131IH]</smiles>

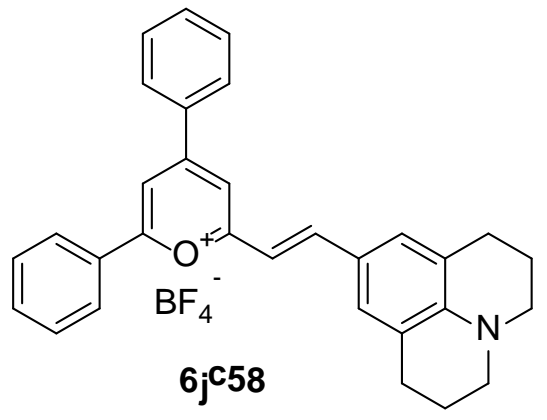


To pyrylium salt 6jc26 (120 mg, $0.37 \mathrm{mmol}$ ) and 9-CHO-julolidine (34 mg, $0.22 \mathrm{mmol}$ ) in $\mathrm{MeOH}$ $(8 \mathrm{~mL})$ was stirred at room temperature overnight. The solvent was removed and the residue was suspended in ether and filtered to give solid. Recrystallization from EtOH gave purple solid of 96 mg.

$\delta_{\mathrm{H}}\left(\mathrm{DMSO}-d_{6}, 400 \mathrm{MHz}\right)$ 8.44-8.14 (m, $\left.7 \mathrm{H}, \mathrm{Ar}, \mathrm{HC}=\right), 7.75-7.60$ (m, $\left.6 \mathrm{H}, \mathrm{Ar}\right), 7.47$ (s, $2 \mathrm{H}, \mathrm{Ar}$ ),

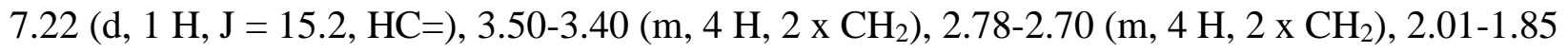
(m, $\left.4 \mathrm{H}, 2 \times \mathrm{CH}_{2}\right)$.

(E)-2-(4-(dimethylamino)styryl)-4,6-bis(3,4-dimethylphenyl)pyrylium boron tetrafluoride salt

\section{6jc59-1}
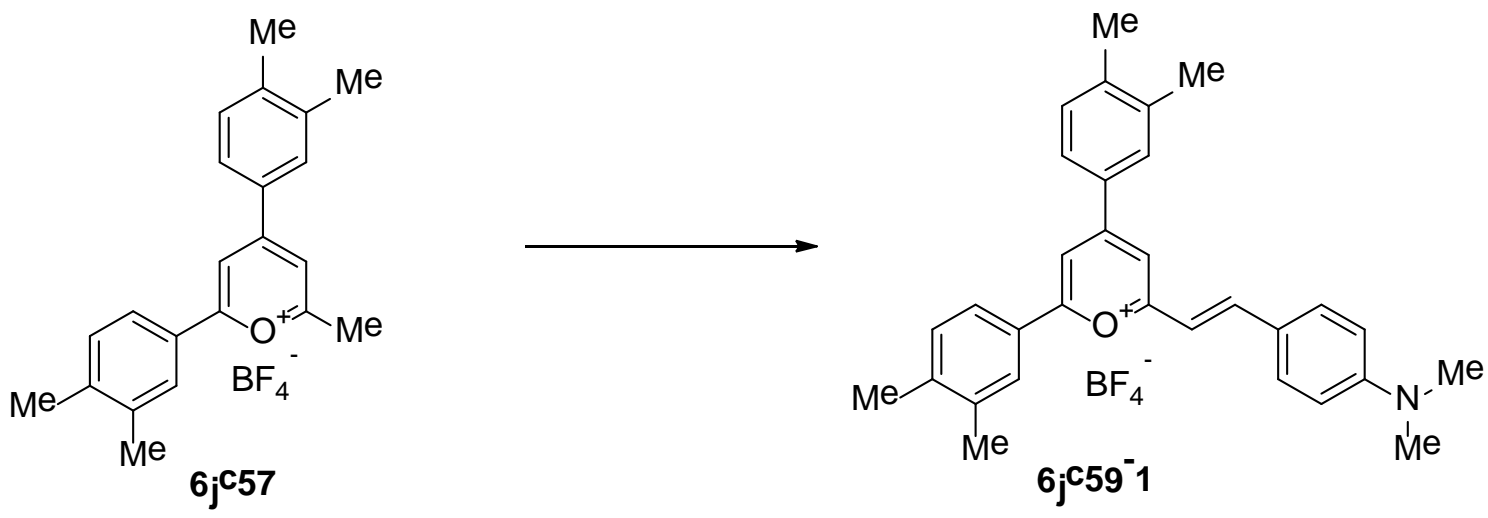

To pyrylium salt 6jc57 (100 mg, $0.22 \mathrm{mmol}$ ) and p-dimethylamino benzaldehyde (34 mg, 0.22 $\mathrm{mmol})$ in $\mathrm{MeOH}(8 \mathrm{~mL})$ was stirred at room temperature overnight. The solvent was removed and the residue was suspended in ether and filtered to give blue solid. Recrystallization from EtOH gave green solid of $79 \mathrm{mg}$.

$\delta_{\mathrm{H}}\left(\mathrm{DMSO}-d_{6}, 400 \mathrm{MHz}\right) 8.53$ (s, $\left.1 \mathrm{H}, \mathrm{Ar}\right), 8.41$ (s, $\left.1 \mathrm{H}, \mathrm{Ar}\right), 8.36$ (d, $\left.1 \mathrm{H}, \mathrm{J}=15.6, \mathrm{HC}=\right), 8.26$ (s, $1 \mathrm{H}, \mathrm{Ar}), 8.23$ (d, $1 \mathrm{H}, \mathrm{J}=$ 8.8, Ar), 8.16 (s, $1 \mathrm{H}, \operatorname{Ar}$ ), 8.12 (d, $1 \mathrm{H}, \mathrm{J}=$ 7.6, Ar), 7.83 (d, $2 \mathrm{H}, \mathrm{J}=$ 8.8, Ar), 7.52-7.43 (m, 2 H, Ar), 7.37 (d, 1 H, J = 15.6, HC=), 6.91 (d, 2 H, J = 8.8, Ar), 3.15 (s, 6 H, NMe 2 ), 2.43 (s, $3 \mathrm{H}, \mathrm{Me}$ ), 2.42 (s, $3 \mathrm{H}, \mathrm{Me}$ ), 2.40 (s, $6 \mathrm{H}, 2$ x Me). 
2-((1E,3E)-4-(4-(dimethylamino)phenyl)buta-1,3-dien-1-yl)-4,6-bis(3,4-

dimethylphenyl)pyrylium boron tetrafluoride salt 6jc59-2<smiles>Cc1cc(-c2ccc(C)c(C)c2)cc(-c2ccc(C)c(C)c2)[o+]1</smiles><smiles>Cc1ccc(-c2cc(/C=C/C=C/c3ccc(N(C)C)cc3)[o+]c(-c3ccc(C)c(C)c3)c2)cc1C</smiles>

To pyrylium salt $6 \mathbf{j c 5 7}$ (100 mg, $0.22 \mathrm{mmol}$ ) and p-dimethylamino cinnamaldehyde $39 \mathrm{mg}, 0.22$ $\mathrm{mmol})$ in $\mathrm{MeOH}(8 \mathrm{~mL})$ was stirred at room temperature overnight. The solvent was removed and the residue was suspended in ether and filtered to give blue solid. Recrystallization from EtOH gave green solid of $70 \mathrm{mg}$.

$\delta_{\mathrm{H}}\left(\mathrm{DMSO}-d_{6}, 400 \mathrm{MHz}\right) 8.58$ (s, $\left.1 \mathrm{H}, \mathrm{Ar}\right), 8.42$ (s, $\left.1 \mathrm{H}, \mathrm{Ar}\right), 8.30-8.16$ (m, $\left.4 \mathrm{H}, \mathrm{Ar}, \mathrm{HC}=\right), 8.14$ (d, $1 \mathrm{H}, \mathrm{J}=$ 8.0, Ar), 7.62 (d, $2 \mathrm{H}, \mathrm{J}=$ 8.4, Ar), 7.53-7.46 (m, $3 \mathrm{H}, \mathrm{Ar}, \mathrm{HC}=$ ), 7.25 (t, $1 \mathrm{H}, \mathrm{J}=14.0$, $\mathrm{HC}=$ ), 6.90-6.77 (m, $3 \mathrm{H}, \mathrm{Ar}, \mathrm{HC}=$ ), 3.07 (s, $6 \mathrm{H}, \mathrm{NMe}_{2}$ ), 2.44 (s, $3 \mathrm{H}, \mathrm{Me}$ ), 2.41 (s, $3 \mathrm{H}, \mathrm{Me}$ ), 2.40 (s, $6 \mathrm{H}, 2$ x Me).

(E)-2-(2-(1H-indol-3-yl)vinyl)-4,6-bis(3,4-dimethylphenyl)pyrylium boron tetrafluoride salt

\section{6jc59-3}<smiles>Cc1cc(-c2ccc(C)c(C)c2)cc(-c2ccc(C)c(C)c2)[o+]1</smiles>
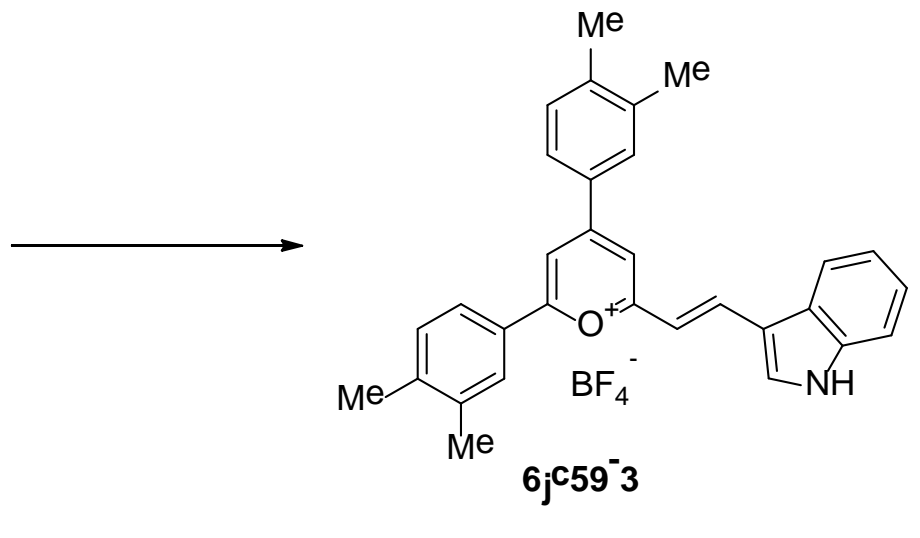

To pyrylium salt 6jc57 (100 mg, $0.22 \mathrm{mmol}$ ) and indole-3-carboxaldehyde (32 mg, $0.22 \mathrm{mmol}$ ) in $\mathrm{MeOH}(8 \mathrm{~mL})$ was stirred at room temperature for 2 days. The solvent was removed and the 
residue was suspended in ether and filtered to give blue solid. Recrystallization from EtOH gave green solid of $55 \mathrm{mg}$.

$\delta_{\mathrm{H}}\left(\mathrm{DMSO}_{6}, 400 \mathrm{MHz}\right) 12.54$ (br s, $\left.1 \mathrm{H}, \mathrm{NH}\right), 8.64$ (d, $\left.1 \mathrm{H}, \mathrm{J}=15.6, \mathrm{HC}=\right), 8.55$ (s, $1 \mathrm{H}, \mathrm{Ar}$ ), 8.52 (s, 1 H, Ar), 8.43 (s, 1 H, Ar), 8.32-8.22 (m, 3 H, Ar), 8.16 (s, 1 H, Ar), 8.12 (d, 1 H, J = 8.4, Ar), 7.63-7.59 (m, 1 H, Ar), 7.56-7.45 (m, 3 H, Ar, HC=), 7.43-7.34 (m, 2 H, Ar), 2.45 (s, 3 H, Me), 2.43 (s, 3 H, Me), 2.41 (s, 6 H, 2 x Me).

(E)-2,4-bis(4-(tert-butyl)phenyl)-6-(4-(dimethylamino)styryl)pyrylium boron tetrafluoride salt 6jc60-1
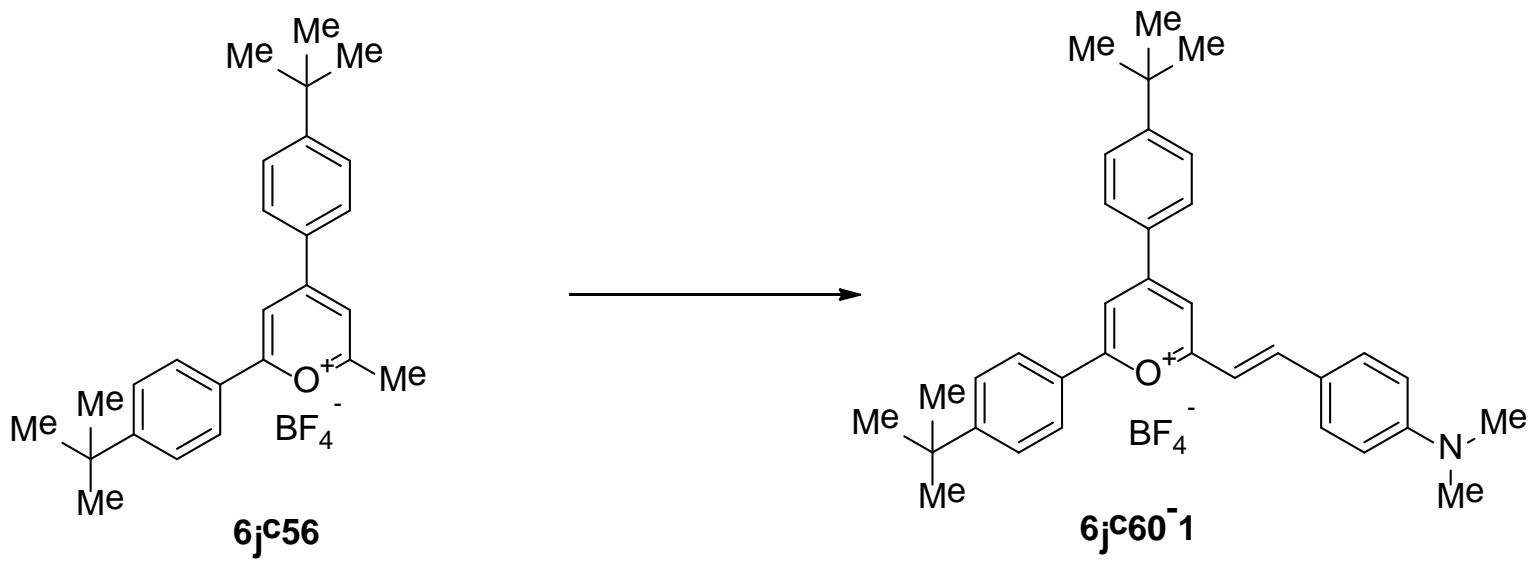

To pyrylium salt $6 \mathbf{j c 5 6}$ (100 mg, $0.22 \mathrm{mmol}$ ) and p-dimethylamino benzaldehyde (40 mg, 0.22 $\mathrm{mmol})$ in $\mathrm{MeOH}(8 \mathrm{~mL})$ was stirred at room temperature overnight. The solvent was removed and the residue was suspended in ether and filtered to give solid. Recrystallization from EtOH gave green solid of $67 \mathrm{mg}$.

$\delta_{\mathrm{H}}\left(\mathrm{DMSO}-d_{6}, 400 \mathrm{MHz}\right) 8.56$ (s, $\left.1 \mathrm{H}, \mathrm{Ar}\right), 8.41$ (s, $\left.1 \mathrm{H}, \mathrm{Ar}\right), 8.40-8.33$ (m, $3 \mathrm{H}, \mathrm{Ar}, \mathrm{HC}=$ ), 8.26 (d, 2 H, J = 8.4, Ar), 7.83 (d, 2 H, J = 9.6, Ar), 7.78-7.71 (m, 4 H, Ar), 7.37 (d, 1 H, J = 15.6, HC=), 6.91 (d, 2 H, J = 8.8, Ar), 3.15 (s, $6 \mathrm{H}, \mathrm{NMe}_{2}$ ), 1.39 (s, $\left.9 \mathrm{H}, \mathrm{tBu}\right), 1.38$ (s, $9 \mathrm{H}, \mathrm{tBu}$ ). 
2,4-bis(4-(tert-butyl)phenyl)-6-((1E,3E)-4-(4-(dimethylamino)phenyl)buta-1,3-dien-1yl)pyrylium boron tetrafluoride salt $\mathbf{6 j} \mathbf{6 0 0 - 2}$
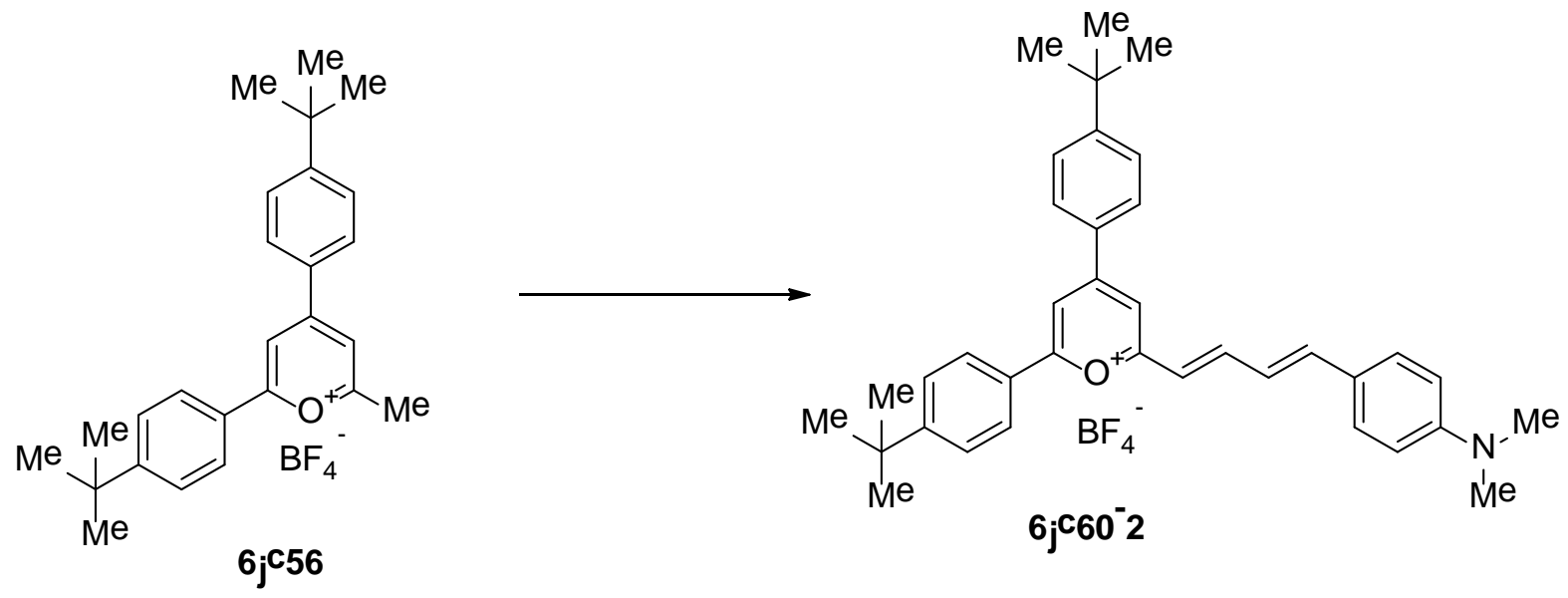

To pyrylium salt $6 \mathbf{j c 5 6}$ (100 mg, $0.22 \mathrm{mmol}$ ) and p-dimethylamino cinnamaldehyde (44 mg, 0.22 $\mathrm{mmol})$ in $\mathrm{MeOH}(8 \mathrm{~mL})$ was stirred at room temperature overnight. The solvent was removed and the residue was suspended in ether and filtered to give solid. Recrystallization from EtOH gave green solid of $50 \mathrm{mg}$.

$\delta_{\mathrm{H}}\left(\mathrm{DMSO}-d_{6}, 400 \mathrm{MHz}\right) 8.61$ (s, $1 \mathrm{H}, \mathrm{Ar}$ ), 8.42-8.37 (m, $3 \mathrm{H}, \mathrm{Ar}$ ), 8.29 (d, $2 \mathrm{H}, \mathrm{J}=8.8, \mathrm{Ar}$ ), 8.22 (t, $1 \mathrm{H}, \mathrm{J}=14.8, \mathrm{HC}=$ ), 7.54 (d, $4 \mathrm{H}, \mathrm{J}=$ 8.0, Ar), 7.62 (d, $2 \mathrm{H}, \mathrm{J}=$ 9.2, Ar), 7.52 (d, $1 \mathrm{H}, \mathrm{J}=14.8$, $\mathrm{HC}=$ ), 7.27 (t, $1 \mathrm{H}, \mathrm{J}=15.2, \mathrm{HC}=), 6.86(\mathrm{~d}, 1 \mathrm{H}, \mathrm{J}=15.2, \mathrm{HC}=), 6.81$ (d, $2 \mathrm{H}, \mathrm{J}=8.8, \mathrm{Ar}), 3.07$ (s, $6 \mathrm{H}, \mathrm{NMe}_{2}$ ), 1.39 (s, $\left.9 \mathrm{H}, \mathrm{tBu}\right), 1.38$ (s, $9 \mathrm{H}, \mathrm{tBu}$ ).

(E)-2-(2-(1H-indol-3-yl)vinyl)-4,6-bis(4-(tert-butyl)phenyl)pyrylium boron tetrafluoride salt 6jc60-3
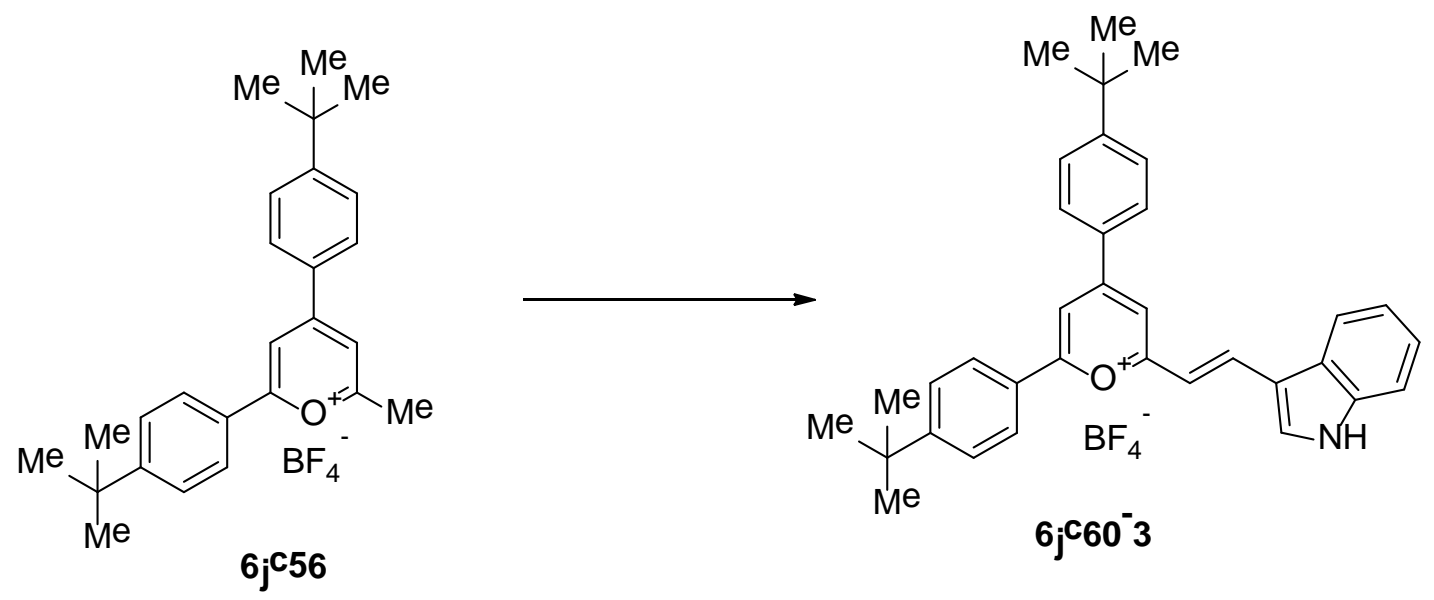

To pyrylium salt 6jc56 (100 mg, $0.22 \mathrm{mmol}$ ) and indole-3-carboxaldehyde (32 mg, $0.22 \mathrm{mmol}$ ) in $\mathrm{MeOH}(8 \mathrm{~mL})$ was stirred at room temperature overnight. The solvent was removed and the 
residue was suspended in ether and filtered to give solid. Recrystallization from EtOH gave green solid of $50 \mathrm{mg}$.

$\delta_{\mathrm{H}}\left(\mathrm{DMSO}-d_{6}, 400 \mathrm{MHz}\right) 12.56$ (br s, $\left.1 \mathrm{H}, \mathrm{NH}\right), 8.67$ (d, $\left.1 \mathrm{H}, \mathrm{J}=15.6, \mathrm{HC}=\right), 8.58$ (s, $1 \mathrm{H}, \mathrm{Ar}$ ), 8.52 (s, 1 H, Ar), 8.46-8.40 (m, 3 H, Ar), 8.32-8.24 (m, 3 H, Ar), 7.80-7.72 (m, 4 H, Ar), 7.66-7.59 (m, 1 H, Ar), 7.51 (d, 1 H, J = 15.6, HC=), 7.43-7.35 (m, 2 H, Ar), 1.39 (s, 18 H, 2 x tBu).

2,4-bis(4-ethylphenyl)-6-methylpyrylium boron tetrafluoride salt $\mathbf{6 j c 6 1}$

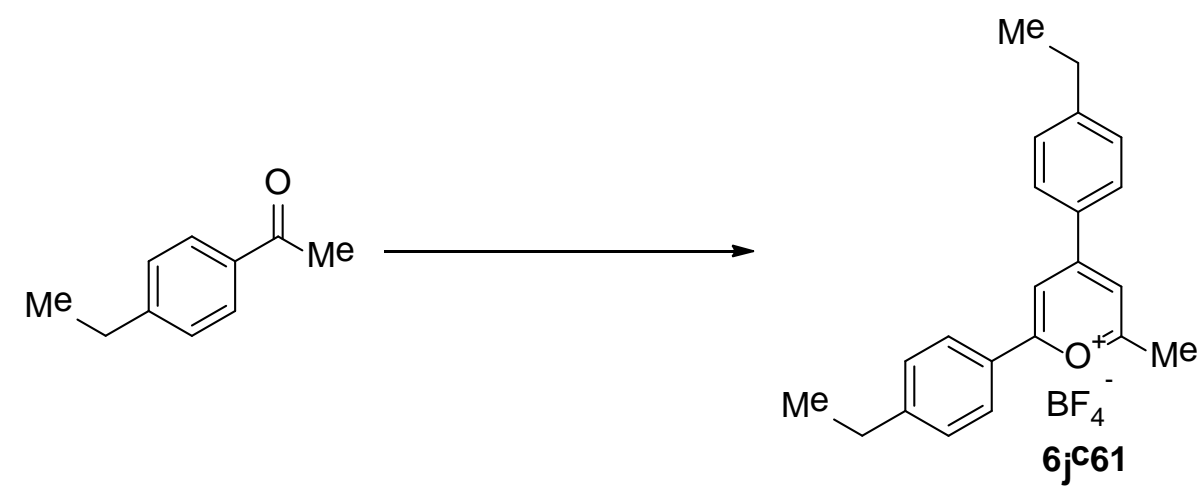

To p-ethyl-acetophenone (2.00 g, $13.51 \mathrm{mmol})$ and acetic anhydride (1.28 mL, $13.51 \mathrm{mmol})$ was added boron trifluoride etherate $(4.17 \mathrm{~mL}, 33.8 \mathrm{mmol})$ at room temperature. The reaction was heated to 135 degrees $\mathrm{C}$ for $3 \mathrm{hr}$, cooled, poured into EtOAc and the yellow solid filtered to give $214 \mathrm{mg}$.

$\delta_{\mathrm{H}}\left(\mathrm{MeOH}-d_{4}, 400 \mathrm{MHz}\right) 8.85$ (s, $1 \mathrm{H}, \mathrm{Ar}$ ), 8.35-8.30 (m, $3 \mathrm{H}, \mathrm{Ar}$ ), 8.24 (d, $\left.2 \mathrm{H}, \mathrm{J}=8.4, \mathrm{Ar}\right), 7.57$ (d, 4 H, J = 8.0, Ar), 2.99 (s, 3 H, Me), 2.81 (q, 4 H, J = 8.0, 2 x CH 2$), 1.30$ (t, $6 \mathrm{H}, \mathrm{J}=8.0,2 \mathrm{x}$ $\left.\mathrm{CH}_{3}\right)$.

(E)-2-(4-(dimethylamino)styryl)-4,6-bis(4-ethylphenyl)pyrylium boron tetrafluoride salt 6jc64-1<smiles>CCc1ccc(-c2cc(C)[o+]c(-c3ccc(CC)cc3)c2)cc1</smiles><smiles>CCc1ccc(-c2cc(/C=C/c3ccc(N(C)C)cc3)[o+]c(-c3ccc(CC)cc3)c2)cc1</smiles> 
To pyrylium salt 6jc61 (50 mg, $0.13 \mathrm{mmol}$ ) and p-dimethylamino benzaldehyde (20 mg, 0.13 $\mathrm{mmol})$ in $\mathrm{MeOH}(8 \mathrm{~mL})$ was stirred at room temperature overnight. The solvent was removed and the residue was suspended in ether and filtered to give blue solid. Recrystallization from EtOH gave brown solid of $41 \mathrm{mg}$.

$\delta_{\mathrm{H}}\left(\mathrm{DMSO}-d_{6}, 400 \mathrm{MHz}\right) 8.56$ (s, $1 \mathrm{H}, \mathrm{Ar}$ ), 8.46-8.33 (m, $\left.4 \mathrm{H}, \mathrm{Ar}, \mathrm{HC}=\right), 8.29$ (d, $2 \mathrm{H}, \mathrm{J}=8.0$, Ar), 7.84 (d, 2 H, J = 8.4, Ar), 7.62-7.54 (m, 4 H, Ar), 7.38 (d, 1 H, J = 16.4, HC=), 6.92 (d, 2 H, $\mathrm{J}=$ 8.4, Ar), 3.15 (s, $6 \mathrm{H}, \mathrm{NMe}_{2}$ ), 2.79 (q, $\left.4 \mathrm{H}, \mathrm{J}=7.2,2 \times \mathrm{CH}_{2}\right), 1.28$ (t, $6 \mathrm{H}, \mathrm{J}=7.2,2 \times \mathrm{CH}_{3}$ ).

2-((1E,3E)-4-(4-(dimethylamino)phenyl)buta-1,3-dien-1-yl)-4,6-bis(4-ethylphenyl)pyrylium boron tetrafluoride salt $\mathbf{6 j} \mathbf{6 6 4 - 2}$
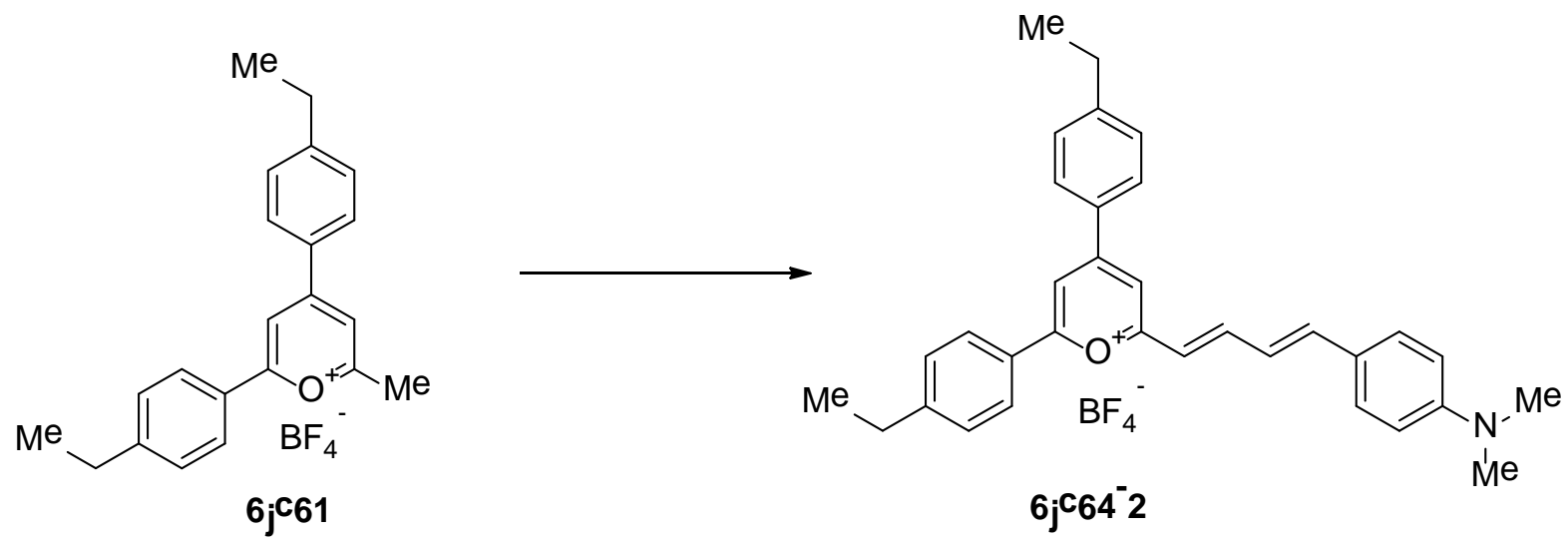

To pyrylium salt $6 \mathbf{j c 6 1}$ (50 mg, $0.13 \mathrm{mmol}$ ) and p-dimethylamino cinnamaldehyde (23 mg, 0.13 $\mathrm{mmol})$ in $\mathrm{MeOH}(8 \mathrm{~mL})$ was stirred at room temperature overnight. The solvent was removed and the residue was suspended in ether and filtered to give blue solid. Recrystallization from EtOH gave brown solid of $31 \mathrm{mg}$.

$\delta_{\mathrm{H}}\left(\mathrm{DMSO}-d_{6}, 400 \mathrm{MHz}\right) 8.63$ (s, $\left.1 \mathrm{H}, \mathrm{Ar}\right), 8.43$ (s, $1 \mathrm{H}, \mathrm{Ar}$ ), 8.40 (d, $2 \mathrm{H}, \mathrm{J}=$ 7.6, Ar), 8.32 (d, 2 $\mathrm{H}, \mathrm{J}=$ 7.6, Ar), 8.24 (t, $1 \mathrm{H}, \mathrm{J}=14.0, \mathrm{HC}=)$, 7.67-7.56 (m, $6 \mathrm{H}, \mathrm{Ar}$ ), 7.52 (d, $1 \mathrm{H}, \mathrm{J}=14.8, \mathrm{HC}=$ ), 7.27 (t, $1 \mathrm{H}, \mathrm{J}=14.8, \mathrm{HC}=), 6.86$ (d, $1 \mathrm{H}, \mathrm{J}=16.0, \mathrm{HC}=), 6.81$ (d, $2 \mathrm{H}, \mathrm{J}=8.8, \mathrm{Ar}$ ), 3.08 (s, $6 \mathrm{H}$, $\mathrm{NMe}_{2}$ ), 2.79 (q, $4 \mathrm{H}, \mathrm{J}=7.2,2 \times \mathrm{CH}_{2}$ ), 1.27 (t, $6 \mathrm{H}, \mathrm{J}=7.2,2 \times \mathrm{CH}_{3}$ ). 
(E)-2-(2-(1H-indol-3-yl)vinyl)-4,6-bis(4-ethylphenyl)pyrylium boron tetrafluoride salt 6jc64-3
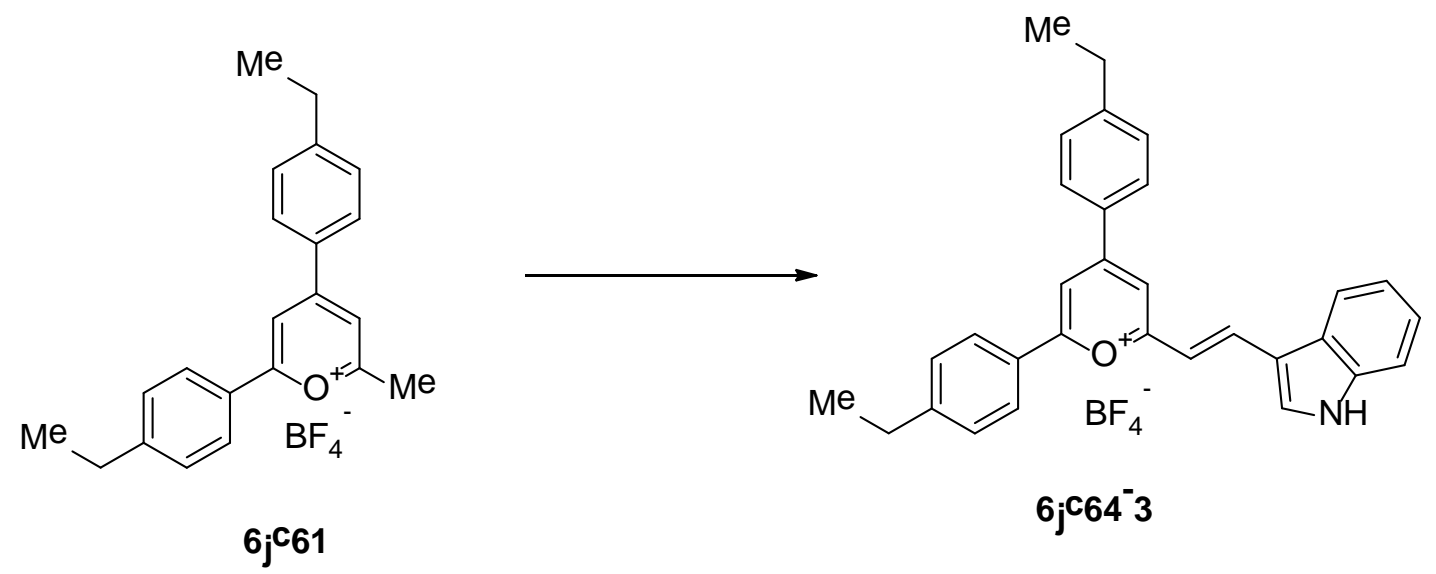

To pyrylium salt $\mathbf{6 j c 6 1}$ (50 mg, $0.13 \mathrm{mmol}$ ) and indole-3-carboxaldehyde (19 mg, $0.13 \mathrm{mmol}$ ) in $\mathrm{MeOH}(8 \mathrm{~mL})$ was stirred at room temperature for 2 days. The solvent was removed and the residue was suspended in ether and filtered to give blue solid. Recrystallization from EtOH gave brown solid of $24 \mathrm{mg}$.

$\delta_{\mathrm{H}}\left(\mathrm{DMSO}-d_{6}, 400 \mathrm{MHz}\right) 12.56$ (br s, $\left.1 \mathrm{H}, \mathrm{NH}\right), 8.68$ (d, $\left.1 \mathrm{H}, \mathrm{J}=15.6, \mathrm{HC}=\right), 8.59$ (s, $1 \mathrm{H}, \mathrm{Ar}$ ), 8.54 (s, 1 H, Ar), 8.47-8.41 (m, 3 H, Ar), 8.34-8.24 (m, 3 H, Ar), 7.66-7.57 (m, 5 H, Ar), 7.51 (d, $1 \mathrm{H}, \mathrm{J}=15.6, \mathrm{HC}=$ ), 7.43-7.37 (m, $2 \mathrm{H}, \mathrm{Ar}$ ), 2.80 (q, $4 \mathrm{H}, \mathrm{J}=8.0,2 \times \mathrm{CH}_{2}$ ), 1.28 (t, $6 \mathrm{H}, \mathrm{J}=8.0$, $\left.2 \times \mathrm{CH}_{3}\right)$.

(E)-2-(2-(1,2,3,5,6,7-hexahydropyrido[3,2,1-ij]quinolin-9-yl)vinyl)-4,6-di-p-tolylpyrylium boron tetrafluoride salt $\mathbf{6 j} \mathbf{6} \mathbf{6 5 - 1}$
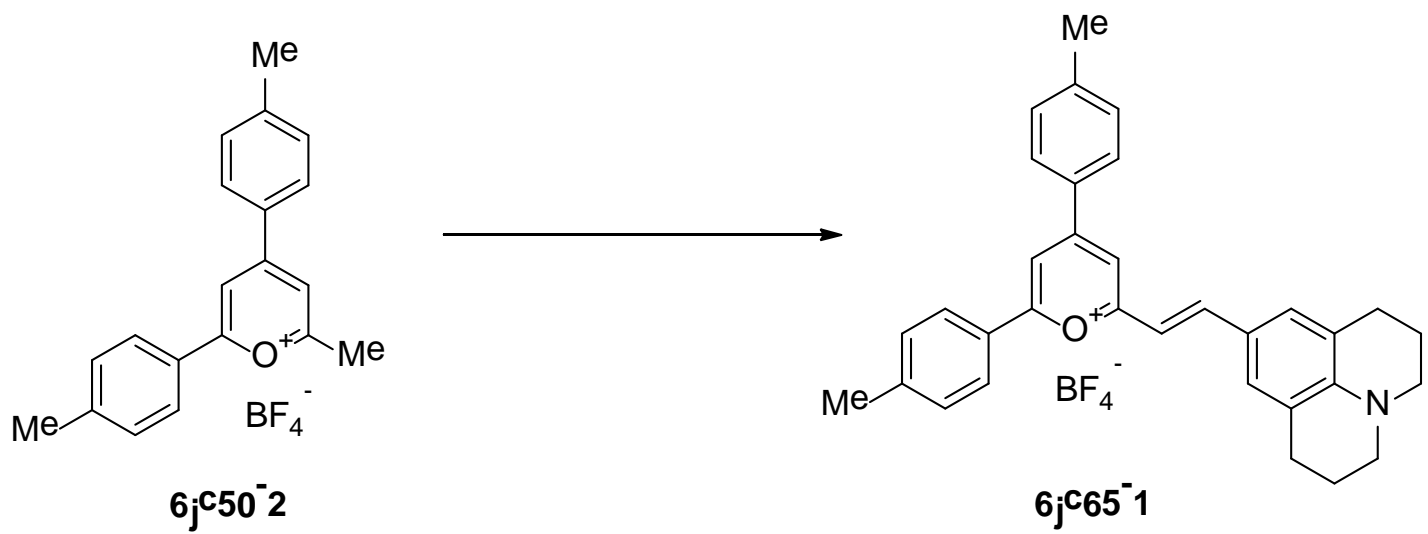

To pyrylium salt 6jc50-2 (50 mg, $0.14 \mathrm{mmol}$ ) and 9-CHO-julolidine (28 mg, $0.14 \mathrm{mmol}$ ) in $\mathrm{MeOH}$ $(8 \mathrm{~mL})$ was stirred at room temperature overnight. The solvent was removed and the residue was suspended in ether and filtered to give solid. Recrystallization from EtOH gave red solid of $48 \mathrm{mg}$. 
$\delta_{\mathrm{H}}\left(\mathrm{DMSO}_{6}, 400 \mathrm{MHz}\right)$ 8.37-8.27 (m, $\left.3 \mathrm{H}, \mathrm{Ar}, \mathrm{HC}=\right)$, 8.21-8.15 (m, $\left.4 \mathrm{H}, \mathrm{Ar}\right), 7.54-7.46$ (m, 4 H, Ar), 7.41 (s, 2 H, Ar), 7.17 (d, 1 H, J = 15.6, HC=), 3.43-3.3.38 (m, $4 \mathrm{H}, 2$ x CH 2 ), 2.79-2.70 (m, $4 \mathrm{H}, 2 \times \mathrm{CH}_{2}$ ), 1.96-1.86 (m, $4 \mathrm{H}, 2 \times \mathrm{CH}_{2}$ ).

(E)-2-(2-(1-methyl-1H-indol-3-yl)vinyl)-4,6-di-p-tolylpyrylium boron tetrafluoride salt 6jc65-2

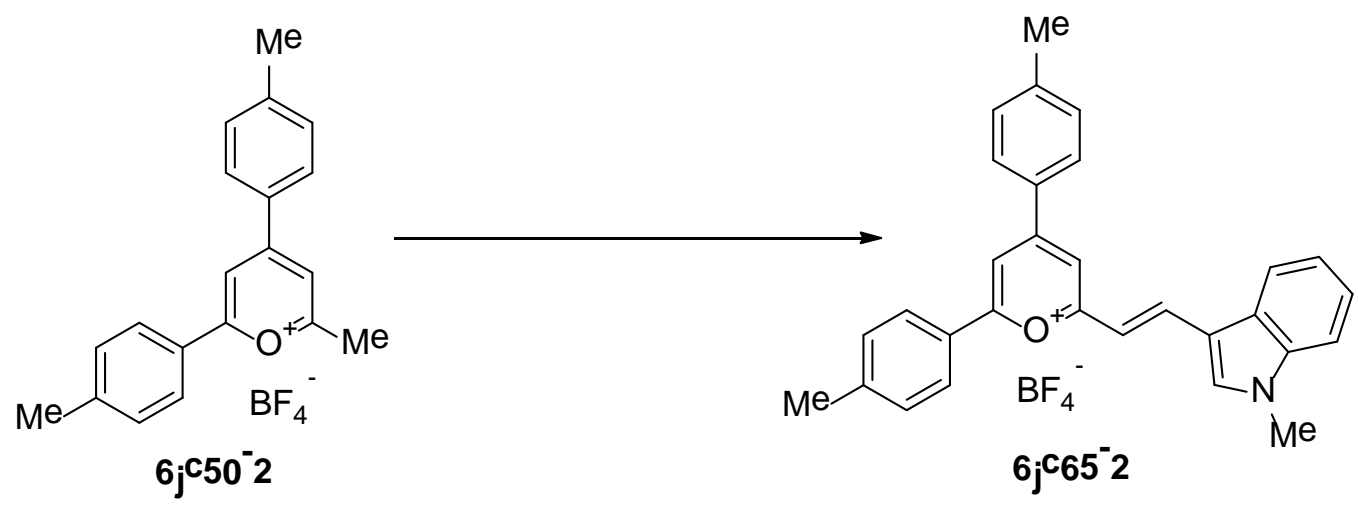

To pyrylium salt 6jc50-2 (50 mg, $0.14 \mathrm{mmol}$ ) and N-methyl-indole-3-carboxaldehyde (23 mg, $0.14 \mathrm{mmol}$ ) in $\mathrm{MeOH}(8 \mathrm{~mL}$ ) was stirred at room temperature for 2 days. The solvent was removed and the residue was suspended in ether and filtered to give blue solid. Recrystallization from EtOH gave purple solid of $40 \mathrm{mg}$.

$\delta_{\mathrm{H}}\left(\mathrm{DMSO}-d_{6}, 400 \mathrm{MHz}\right) 8.64$ (d, $1 \mathrm{H}, \mathrm{J}=15.6, \mathrm{HC}=$ ), 8.57 (s, $1 \mathrm{H}, \mathrm{Ar}$ ), 8.53 (s, $1 \mathrm{H}, \mathrm{Ar}$ ), 8.458.37 (m, 3 H, Ar), 8.32-8.23 (m, 3 H, Ar), 7.70 (d, 1 H, J = 6.8, Ar), 7.56 (t, 4 H, J = 6.8, Ar), (m, 5 H, Ar), 7.50-7.40 (m, $3 \mathrm{H}, \mathrm{Ar}, \mathrm{HC}=), 3.97$ (s, $3 \mathrm{H}, \mathrm{NMe}$ ), 2.50 (s, $6 \mathrm{H}, 2$ x $\mathrm{CH}_{3}$ ).

(E)-2-(2-(1-methyl-1H-indol-3-yl)vinyl)-4,6-diphenylpyrylium boron tetrafluoride salt 6jc66-1<smiles>Cc1cc(-c2ccccc2)cc(-c2ccccc2)[o+]1</smiles><smiles>Cn1cc(/C=C/c2cc(-c3ccccc3)cc(-c3ccccc3)[o+]2)c2ccccc21</smiles>

$6 \mathrm{j}_{66}{ }^{-} 1$

To pyrylium salt 6jc26 (53 mg, $0.16 \mathrm{mmol}$ ) and N-methyl-indole-3-carboxaldehyde ( $25 \mathrm{mg}, 0.16$ $\mathrm{mmol})$ in $\mathrm{MeOH}(8 \mathrm{~mL})$ was stirred at room temperature for 2 days. The solvent was removed and the residue was suspended in ether and filtered to give blue solid. Recrystallization from EtOH gave purple solid of $34 \mathrm{mg}$. 
$\delta_{\mathrm{H}}\left(\mathrm{DMSO}_{6}, d_{6}, 400 \mathrm{MHz}\right) 8.72(\mathrm{~d}, 1 \mathrm{H}, \mathrm{J}=15.6, \mathrm{HC}=), 8.63$ (s, $\left.1 \mathrm{H}, \mathrm{Ar}\right), 8.59$ (s, $\left.1 \mathrm{H}, \mathrm{Ar}\right), 8.53$ (d, 2 H, J = 7.6, Ar), 8.45 (s, 1 H, Ar), 8.38-8.29 (m, 3 H, Ar), 7.84-7.70 (m, 7 H, Ar), 7.52 (d, 1 $\mathrm{H}, \mathrm{J}=15.6, \mathrm{HC}=$ ), 7.49-7.43 (m, $2 \mathrm{H}, \mathrm{Ar}$ ), 3.99 (s, $3 \mathrm{H}, \mathrm{NMe}$ ).

(E)-2,4-bis(4-ethylphenyl)-6-(2-(1-methyl-1H-indol-3-yl)vinyl)pyrylium boron tetrafluoride salt 6jc66-2
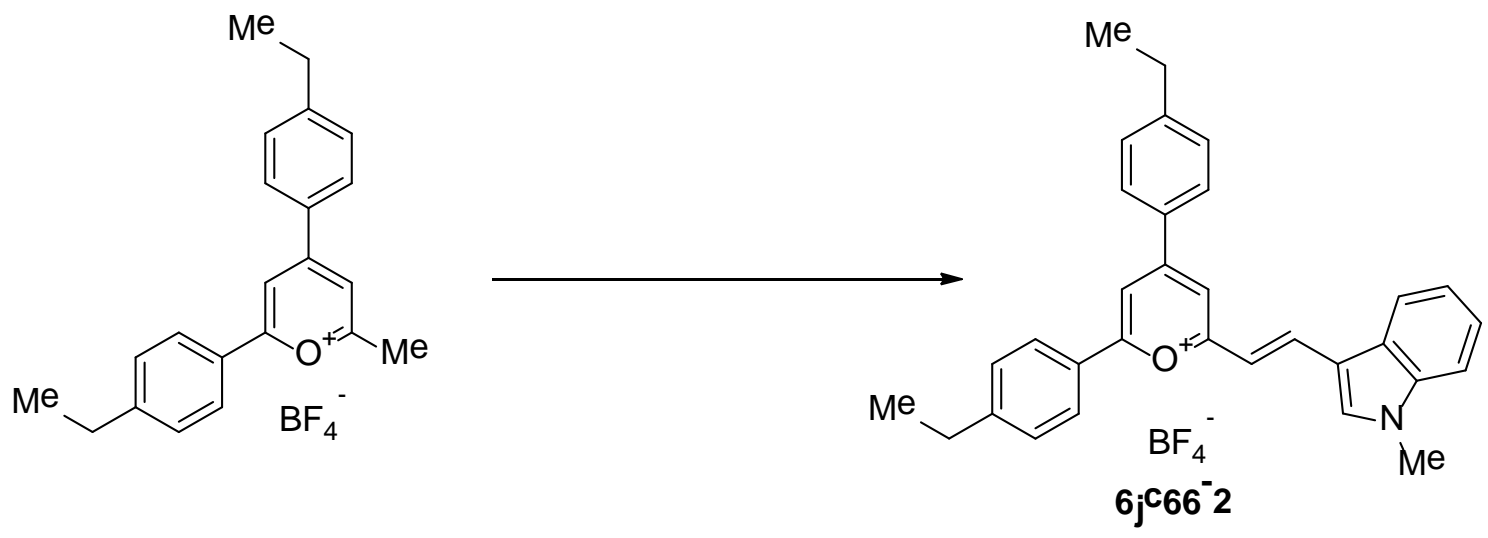

To pyrylium salt 6jc61 (62 mg, 0.16 mmol) and N-methyl-indole-3-carboxaldehyde (25 mg, 0. 16 $\mathrm{mmol})$ in $\mathrm{MeOH}(8 \mathrm{~mL})$ was stirred at room temperature for 2 days. The solvent was removed and the residue was suspended in ether and filtered to give blue solid. Recrystallization from EtOH gave purple solid of $13 \mathrm{mg}$.

$\delta_{\mathrm{H}}\left(\mathrm{DMSO}-d_{6}, 400 \mathrm{MHz}\right) 8.65$ (d, $\left.1 \mathrm{H}, \mathrm{J}=15.6, \mathrm{HC}=\right), 8.59$ (s, $1 \mathrm{H}, \mathrm{Ar}$ ), 8.54 (s, $\left.1 \mathrm{H}, \mathrm{Ar}\right), 8.45$ (d, 2 H, J = 7.6, Ar), 8.41 (s, 1 H, Ar), 8.34-8.26 (m, 3 H, Ar), 7.74-7.68 (m, 1 H, Ar), 7.64-7.56 (m, 4 H, Ar), 7.52-7.42 (m, 3 H, Ar), 3.98 (s, 3 H, NMe), 3.00-2.75 (m, 4 H, 2 x CH 2$), 1.29$ (t, 6 $\left.\mathrm{H}, \mathrm{J}=7.2,2 \times \mathrm{CH}_{3}\right)$. 
(E)-2,4-bis(4-(tert-butyl)phenyl)-6-(2-(1-methyl-1H-indol-3-yl)vinyl)pyrylium boron tetrafluoride salt $\mathbf{6 j} \mathbf{j} \mathbf{6 6 - 3}$
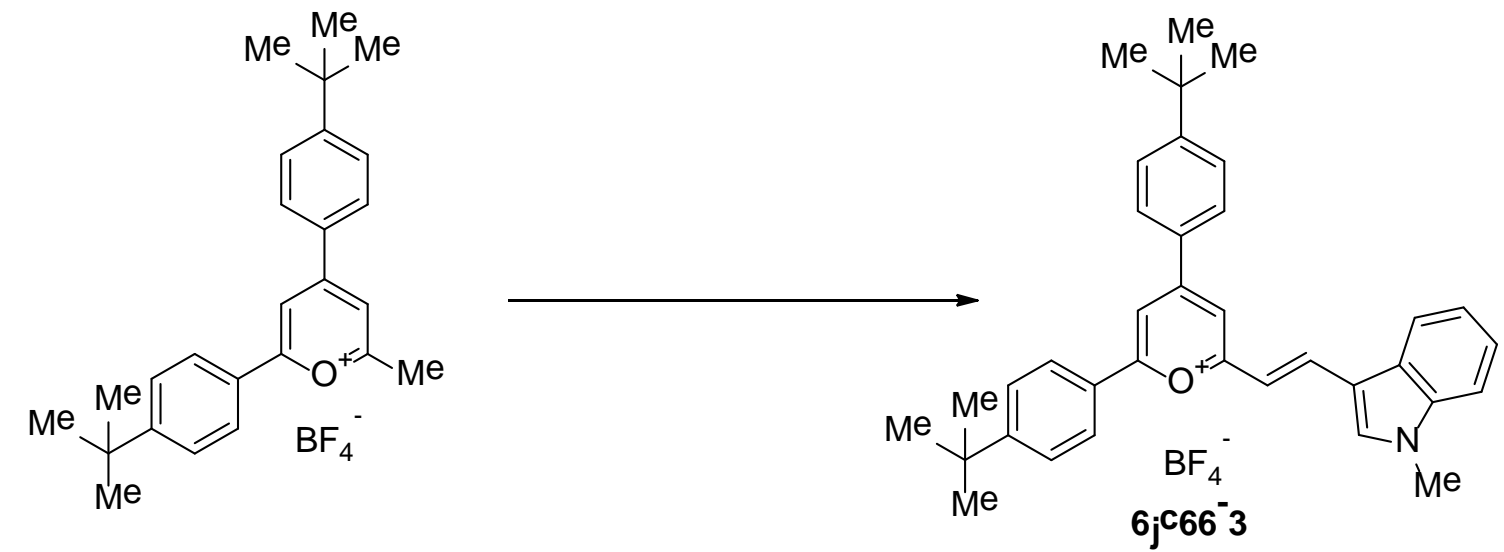

To pyrylium salt $6 \mathbf{j c 5 6}$ (71 mg, $0.16 \mathrm{mmol}$ ) and N-methyl-indole-3-carboxaldehyde ( $25 \mathrm{mg}, 0.16$ $\mathrm{mmol})$ in $\mathrm{MeOH}(8 \mathrm{~mL})$ was stirred at room temperature for 2 days. The solvent was removed and the residue was suspended in ether and filtered to give blue solid. Recrystallization from EtOH gave purple solid of $27 \mathrm{mg}$.

$\delta_{\mathrm{H}}\left(\mathrm{DMSO}_{-} \mathrm{d}_{6}, 400 \mathrm{MHz}\right) 8.64$ (d, $\left.1 \mathrm{H}, \mathrm{J}=15.6, \mathrm{HC}=\right), 8.58$ (s, $\left.1 \mathrm{H}, \mathrm{Ar}\right), 8.52(\mathrm{~s}, 1 \mathrm{H}, \mathrm{Ar}), 8.47-$ 8.40 (m, 3 H, Ar), 8.32-8.24 (m, 3 H, Ar), 7.81-7.68 (m, 5 H, Ar), 7.53-7.42 (m, 3 H, Ar, HC=), 3.99 (s, $3 \mathrm{H}, \mathrm{NMe}), 1.40$ (s, $18 \mathrm{H}, 6$ x CH 3 ).

(E)-2,4-bis(3,4-dimethylphenyl)-6-(2-(1-methyl-1H-indol-3-yl)vinyl)pyrylium boron tetrafluoride salt 6jc66-4

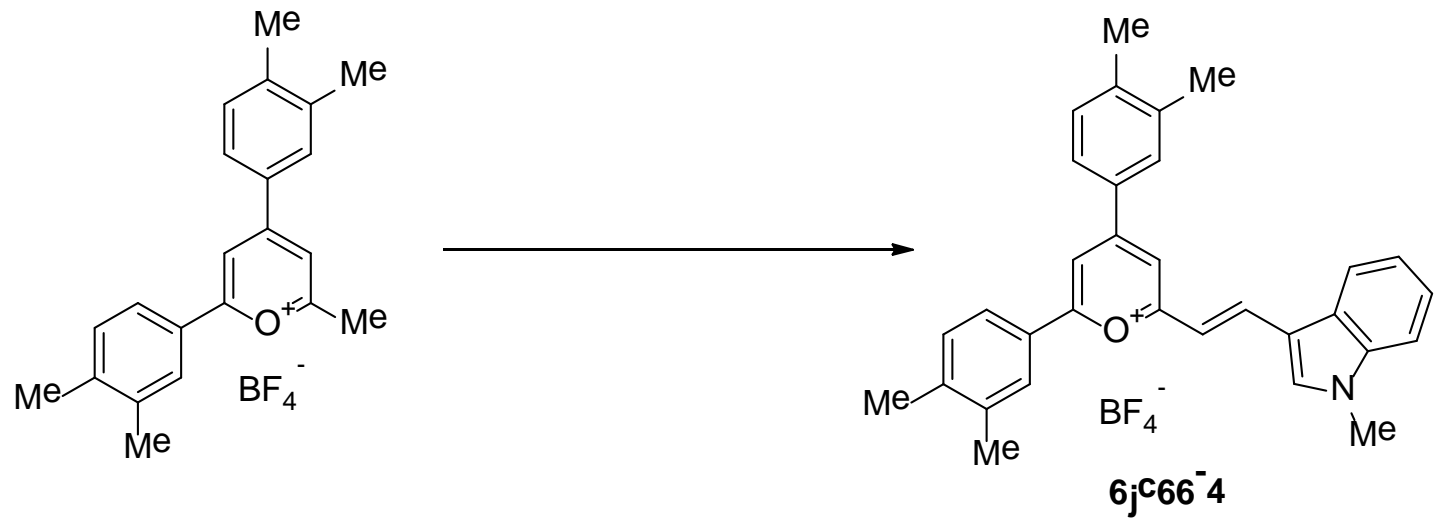

To pyrylium salt 6jc57 (67 mg, $0.16 \mathrm{mmol}$ ) and N-methyl-indole-3-carboxaldehyde ( $25 \mathrm{mg}, 0.16$ $\mathrm{mmol})$ in $\mathrm{MeOH}(8 \mathrm{~mL})$ was stirred at room temperature for 2 days. The solvent was removed and the residue was suspended in ether and filtered to give blue solid. Recrystallization from EtOH gave green solid of $38 \mathrm{mg}$. 
$\delta_{\mathrm{H}}\left(\mathrm{DMSO}-\mathrm{d}_{6}, 400 \mathrm{MHz}\right) 8.61$ (d, $\left.1 \mathrm{H}, \mathrm{J}=16.0, \mathrm{HC}=\right)$, 8.54 (s, $\left.1 \mathrm{H}, \mathrm{Ar}\right), 8.51$ (s, $\left.1 \mathrm{H}, \mathrm{Ar}\right), 8.40$ (s, $1 \mathrm{H}, \mathrm{Ar}$ ), 8.34-8.8.22 (m, $3 \mathrm{H}, \mathrm{Ar}$ ), 8.19-8.8.06 (m, $2 \mathrm{H}, \mathrm{Ar}$ ), 7.70 (d, $1 \mathrm{H}, \mathrm{J}=$ 6.8, Ar), 7.58-7.40 (m, $5 \mathrm{H}, \mathrm{Ar}, \mathrm{HC}=$ ), 3.97 (s, $3 \mathrm{H}, \mathrm{NMe}), 2.45$ (s, $3 \mathrm{H}, \mathrm{CH}_{3}$ ), 2.43 (s, $\left.3 \mathrm{H}, \mathrm{CH}_{3}\right), 2.41$ (s, $6 \mathrm{H}, 2 \mathrm{x}$ $\left.\mathrm{CH}_{3}\right)$.

2,4-diphenyl-6-((1E,3E)-3-(1,3,3-trimethylindolin-2-ylidene)prop-1-en-1-yl)pyrylium boron tetrafluoride salt $\mathbf{6 j} \mathbf{j} \mathbf{6 7}$<smiles>C[C+]1C=C(c2ccccc2)C=C(c2ccccc2)[O+]1</smiles><smiles>CN1/C(=C/C=C/c2cc(-c3ccccc3)cc(-c3ccccc3)[o+]2)C(C)(C)c2ccccc21</smiles>

To pyrylium salt 6jc26 (50 mg, $0.14 \mathrm{mmol}$ ) and 2-(1,3,3-Trimethylindolin-2-ylidene)acetaldehyde (28 mg, $0.14 \mathrm{mmol}$ ) in acetic anhydride ( $2 \mathrm{~mL}$ ) was stirred at reflux for $1 \mathrm{hr}$. The reaction was cooled and co- evaporated with toluene (x 3), washed with ether and decanted (x 2). The residue was purified by column chromatography DCM/MeOH and transferred to a vial to give a blue solid $69 \mathrm{mg}$.

$\delta_{\mathrm{H}}\left(\mathrm{DMSO}-d_{6}, 400 \mathrm{MHz}\right) 8.47$ (t, $\left.1 \mathrm{H}, \mathrm{J}=13.2, \mathrm{HC}=\right)$ ), 8.26-8.10 (m, $\left.4 \mathrm{H}, \mathrm{Ar}\right), 7.95$ (s, $1 \mathrm{H}, \mathrm{Ar}$ ), 7.90-7.59 (m, 8 H, Ar), 7.53-7.44 (m, 2 H, Ar), 7.35 (t, 1 H, J = 7.6, Ar), 6.58 (d, $1 \mathrm{H}, \mathrm{J}=13.2$, $\mathrm{HC}=$ ), 6.39 (br s, $1 \mathrm{H}, \mathrm{HC}=$ ), 3.73 (s, $3 \mathrm{H}, \mathrm{NMe}$ ), 1.74 (s, $6 \mathrm{H}, 2$ x $\mathrm{CH}_{3}$ ).

2,4-di-p-tolyl-6-((1E,3E)-3-(1,3,3-trimethylindolin-2-ylidene)prop-1-en-1-yl)pyrylium boron tetrafluoride salt $\mathbf{6 j} \mathbf{6} \mathbf{6 7 - A}$<smiles>Cc1ccc(-c2cc(C)[o+]c(-c3ccc(C)cc3)c2)cc1</smiles><smiles>Cc1ccc(-c2cc(/C=C/C=C3/N(C)c4ccccc4C3(C)C)[o+]c(-c3ccc(C)cc3)c2)cc1</smiles>

$6 \mathrm{j} 677^{-} \mathrm{A}$ 
To pyrylium salt $\mathbf{6 j c 5 0 - 2} \quad(50 \quad \mathrm{mg}, \quad 0.14 \quad \mathrm{mmol}$ ) and 2-(1,3,3-Trimethylindolin-2ylidene)acetaldehyde (28 mg, $0.14 \mathrm{mmol}$ ) in acetic anhydride $(2 \mathrm{~mL})$ was stirred at reflux for 30 mins. The reaction was cooled and co evaporated with toluene (x 3), washed with ether and decanted (x 2). The blue solid was transferred to a vial to give $71 \mathrm{mg}$.

$\delta_{\mathrm{H}}\left(\mathrm{DMSO}-d_{6}, 400 \mathrm{MHz}\right) 8.41$ (t, $\left.1 \mathrm{H}, \mathrm{J}=14.0, \mathrm{HC}=\right), 8.09$ (d, $\left.1 \mathrm{H}, \mathrm{J}=8.0, \mathrm{Ar}\right), 8.05$ (d, $1 \mathrm{H}, \mathrm{J}=$ 8.0, Ar), 7.91 (s, $1 \mathrm{H}, \mathrm{Ar}), 7.88-7.72$ (m, $1 \mathrm{H}, \mathrm{Ar}), 7.62$ (d, $1 \mathrm{H}, \mathrm{J}=$ 8.0, Ar), 7.51-7.37 (m, $6 \mathrm{H}$, Ar), 7.30-7.24 (m, $1 \mathrm{H}, \mathrm{Ar}$ ), 6.45 (d, $1 \mathrm{H}, \mathrm{J}=14.0, \mathrm{HC}=), 6.35$ (br s, $1 \mathrm{H}, \mathrm{HC}=), 3.65$ (s, $3 \mathrm{H}$, $\mathrm{NMe}$ ), 2.43 (s, $\left.3 \mathrm{H}, \mathrm{ArCH}_{3}\right), 2.40$ (s, $\left.3 \mathrm{H}, \mathrm{ArCH}_{3}\right), 1.69$ (s, $6 \mathrm{H}, 2 \mathrm{x} \mathrm{CH}$ ).

(E)-2,4-bis(4-chlorophenyl)-6-(2-(1-methyl-1H-indol-3-yl)vinyl)pyrylium boron tetrafluoride salt 6jc68-1<smiles>Cc1cc(-c2ccc(Cl)cc2)cc(-c2ccc(Cl)cc2)[o+]1</smiles><smiles>Cn1cc(/C=C/c2cc(-c3ccc(Cl)cc3)cc(-c3ccc(Cl)cc3)[o+]2)c2ccccc21</smiles>

To pyrylium salt 6jc32-1 (52 mg, $0.14 \mathrm{mmol}$ ) and N-methyl-indole-3-carboxaldehyde (23 mg, $0.14 \mathrm{mmol}$ ) in $\mathrm{MeOH}(8 \mathrm{~mL})$ was stirred at room temperature for 2 days. The solvent was removed and the residue was suspended in ether and filtered to give blue solid. Recrystallization from EtOH gave purple solid of $27 \mathrm{mg}$.

$\delta_{\mathrm{H}}\left(\mathrm{DMSO}-d_{6}, 400 \mathrm{MHz}\right) 8.75(\mathrm{~d}, 1 \mathrm{H}, \mathrm{J}=16.0, \mathrm{HC}=), 8.63$ (s, $1 \mathrm{H}, \mathrm{Ar}$ ), 8.60 (s, $1 \mathrm{H}, \mathrm{Ar}$ ), 8.54 (d, 2 H, J = 8.4, Ar), 8.45 (s, 1 H, Ar), 8.40-8.28 (m, 3 H, Ar), 7.88-7.80 (m, 4 H, Ar), 7.76-7.01 (m, 1 H, Ar), 7.55-7.43 (m, 3 H, Ar, HC=), 4.00 (s, 3 H, NMe). 
(E)-2,4-bis(4-bromophenyl)-6-(2-(1-methyl-1H-indol-3-yl)vinyl)pyrylium boron tetrafluoride salt 6jc68-2<smiles>Cc1cc(-c2ccc(Br)cc2)cc(-c2ccc(Br)cc2)[o+]1</smiles><smiles>Cn1cc(/C=C/c2cc(-c3ccc(Br)cc3)cc(-c3ccc(Br)cc3)[o+]2)c2ccccc21</smiles>

To pyrylium salt 6jc47 (63 mg, $0.14 \mathrm{mmol}$ ) and N-methyl-indole-3-carboxaldehyde (23 mg, 0.14 $\mathrm{mmol})$ in $\mathrm{MeOH}(8 \mathrm{~mL})$ was stirred at room temperature for 2 days. The solvent was removed and the residue was suspended in ether and filtered to give blue solid. Recrystallization from EtOH gave purple solid of $20 \mathrm{mg}$.

$\delta_{\mathrm{H}}\left(\mathrm{DMSO}-\mathrm{d}_{6}, 400 \mathrm{MHz}\right) 8.74$ (d, $\left.1 \mathrm{H}, \mathrm{J}=16.0, \mathrm{HC}=\right), 8.62$ (s, $\left.1 \mathrm{H}, \mathrm{Ar}\right), 8.61(\mathrm{~s}, 1 \mathrm{H}, \mathrm{Ar}), 8.48-$ 8.42 (m, 3 H, Ar), 8.32-8.23 (m, 3 H, Ar), 8.06-7.92 (m, 4 H, Ar), 7.76-7.00 (m, 1 H, Ar), 7.547.42 (m, 3 H, Ar, HC=), 4.00 (s, 3 H, NMe).

(E)-2,4-bis(4-ethylphenyl)-6-(2-(1,2,3,5,6,7-hexahydropyrido[3,2,1-ij]quinolin-9yl)vinyl)pyrylium boron tetrafluoride salt 6jc69-1<smiles></smiles>

To pyrylium salt 6jc61 (46 mg, $0.12 \mathrm{mmol}$ ) and 9-CHO-julolidine (25 mg, $0.12 \mathrm{mmol}$ ) in $\mathrm{MeOH}$ $(8 \mathrm{~mL})$ was stirred at room temperature overnight. The solvent was removed and the residue was suspended in ether and filtered to give solid. Recrystallization from EtOH gave purple solid of 44 mg. 
$\delta_{\mathrm{H}}\left(\mathrm{DMSO}_{6}, 400 \mathrm{MHz}\right)$ 8.40-8.32 (m, $\left.3 \mathrm{H}, \mathrm{Ar}, \mathrm{HC}=\right)$, 8.27-8.18 (m, $\left.4 \mathrm{H}, \mathrm{Ar}\right), 7.60-7.52$ (m, 4 $\mathrm{H}, \mathrm{Ar}$ ), 7.45 (s, $2 \mathrm{H}, \mathrm{Ar}$ ), 7.21 (d, $1 \mathrm{H}, \mathrm{J}=15.6, \mathrm{HC}=), 3.50-3.40$ (m, $4 \mathrm{H}, 2 \mathrm{x} \mathrm{CH}_{2}$ ), 2.83-2.70 (m, $8 \mathrm{H}, 4 \times \mathrm{CH}_{2}$ ), 1.99-1.87 (m, $4 \mathrm{H}, 2 \times \mathrm{CH}_{2}$ ), 1.27 (t, $6 \mathrm{H}, \mathrm{J}=7.2,2 \times \mathrm{CH}_{3}$ ).

(E)-2,4-bis(4-(tert-butyl)phenyl)-6-(2-(1,2,3,5,6,7-hexahydropyrido[3,2,1-ij]quinolin-9yl)vinyl)pyrylium boron tetrafluoride salt 6jc69-2
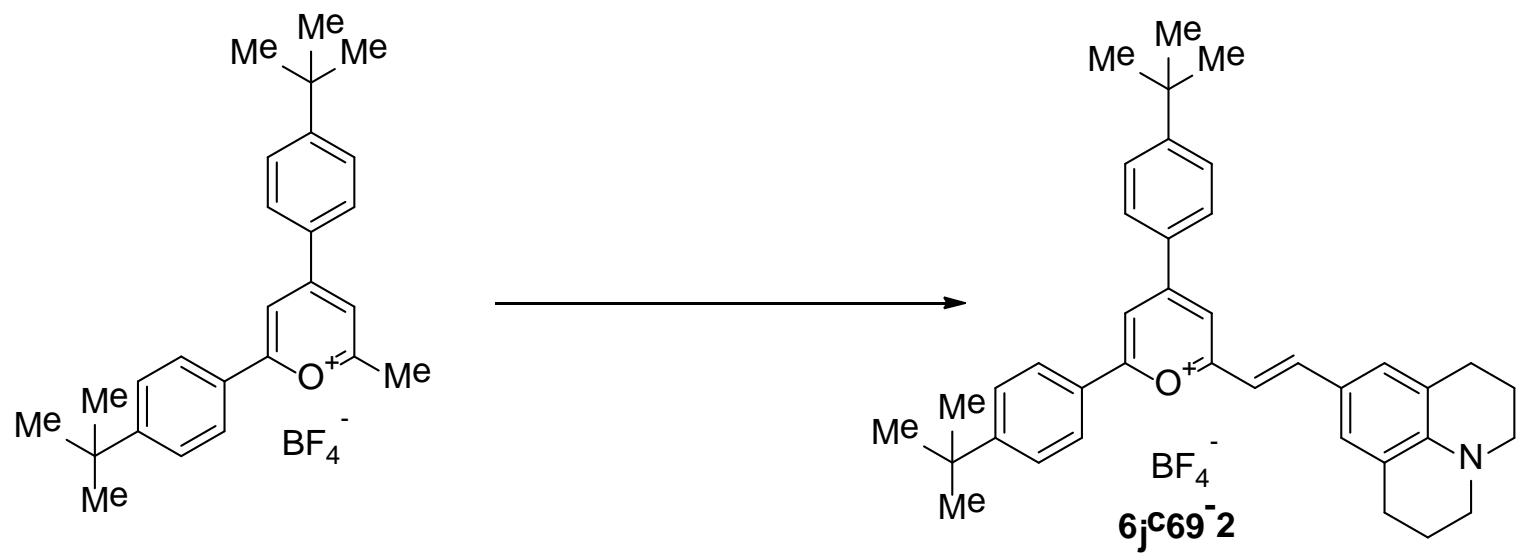

To pyrylium salt 6 jc56 (53 mg, $0.12 \mathrm{mmol}$ ) and 9-CHO-julolidine ( $25 \mathrm{mg}, 0.12 \mathrm{mmol}$ ) in $\mathrm{MeOH}$ $(8 \mathrm{~mL})$ was stirred at room temperature overnight. The solvent was removed and the residue was suspended in ether and filtered to give solid. Recrystallization from EtOH gave purple solid of 74 mg.

$\delta_{\mathrm{H}}\left(\mathrm{DMSO}-d_{6}, 400 \mathrm{MHz}\right)$ 8.38-8.31 (m, $\left.3 \mathrm{H}, \mathrm{Ar}, \mathrm{HC}=\right)$, 8.24-8.15 (m, $\left.4 \mathrm{H}, \mathrm{Ar}\right), 7.78-7.67(\mathrm{~m}, 4$ H, Ar), 7.45 (s, 2 H, Ar), 7.19 (d, 1 H, J = 15.6, HC=), 3.49-3.40 (m, 4 H, $2 x \mathrm{CH}_{2}$ ), 2.80-2.72 (m, $\left.4 \mathrm{H}, 2 \times \mathrm{CH}_{2}\right), 1.97-1.88$ (m, $\left.4 \mathrm{H}, 2 \times \mathrm{CH}_{2}\right), 1.38$ (s, $18 \mathrm{H}, 9 \times \mathrm{CH}_{3}$ ).

(E)-2,4-bis(3,4-dimethylphenyl)-6-(2-(1,2,3,5,6,7-hexahydropyrido[3,2,1-ij]quinolin-9yl)vinyl)pyrylium boron tetrafluoride salt 6jc69-3<smiles>Cc1ccc(-c2cc(-c3ccc(C)c(C)c3)cc(-c3cc(/C=C/c4cc5c6c(c4)CCCN6CCC5)cc(-c4ccc(C)c(C)c4)[o+]3)c2)cc1C</smiles> 
To pyrylium salt 6jc57 (50 mg, $0.12 \mathrm{mmol}$ ) and 9-CHO-julolidine (25 mg, $0.12 \mathrm{mmol}$ ) in $\mathrm{MeOH}$ $(8 \mathrm{~mL})$ was stirred at room temperature overnight. The solvent was removed and the residue was suspended in ether and filtered to give solid. Recrystallization from EtOH gave green solid of 64 mg.

$\delta_{\mathrm{H}}\left(\mathrm{DMSO}-d_{6}, 400 \mathrm{MHz}\right) 8.33$ (s, $\left.1 \mathrm{H}, \mathrm{Ar}\right), 8.25-8.10$ (m, $\left.4 \mathrm{H}, \mathrm{Ar}, \mathrm{HC}=\right), 8.08$ (s, $\left.1 \mathrm{H}, \mathrm{Ar}\right), 8.04$ (d, 1 H, J =7.6, Ar), 7.50-7.43 (m, 2 H, Ar), 7.42 (s, 2 H, Ar), 7.18 (d, 1 H, J = 16.0, HC=), 3.483.40 (m, 4 H, $2 \times \mathrm{CH}_{2}$ ), 2.79-2.70 (m, $4 \mathrm{H}, 2$ x CH 2 ), 2.42 (s, $3 \mathrm{H}, \mathrm{CH}_{3}$ ), 2.40 (s, $\left.3 \mathrm{H}, \mathrm{CH}_{3}\right), 2.38$ (s, $\left.6 \mathrm{H}, 2 \times \mathrm{CH}_{3}\right), 1.99-1.88$ (m, $\left.4 \mathrm{H}, 2 \times \mathrm{CH}_{2}\right)$.

(E)-2,4-bis(4-chlorophenyl)-6-(2-(1,2,3,5,6,7-hexahydropyrido[3,2,1-ij]quinolin-9yl)vinyl)pyrylium boron tetrafluoride salt 6jc69-4

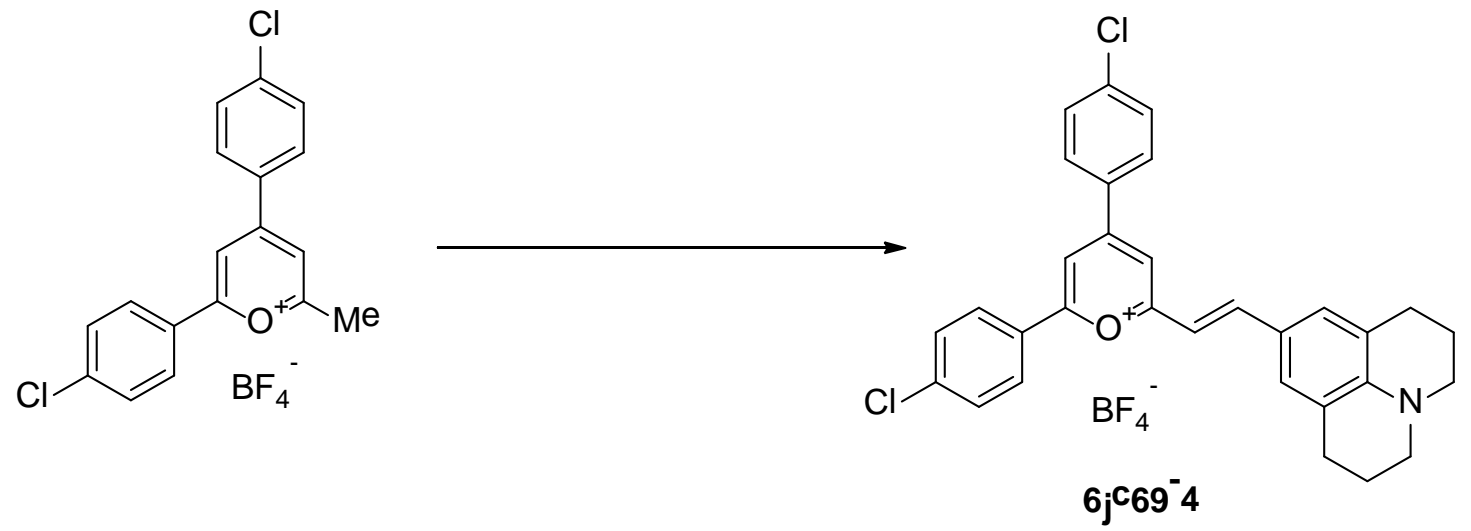

To pyrylium salt 6jc32-1 (48 mg, $0.12 \mathrm{mmol}$ ) and 9-CHO-julolidine (25 mg, $0.12 \mathrm{mmol}$ ) in $\mathrm{MeOH}$ $(8 \mathrm{~mL})$ was stirred at room temperature overnight. The solvent was removed and the residue was suspended in ether and filtered to give solid. Recrystallization from EtOH gave purple solid of 39 mg.

$\delta_{\mathrm{H}}\left(\mathrm{DMSO}-\mathrm{d}_{6}, 400 \mathrm{MHz}\right) 8.40$ (d, $\left.2 \mathrm{H}, \mathrm{J}=8.4, \mathrm{Ar}\right), 8.32$ (s, $\left.1 \mathrm{H}, \mathrm{Ar}\right), 8.30-8.17$ (m, $\left.4 \mathrm{H}, \mathrm{Ar}, \mathrm{HC}=\right)$, 7.83-7.51 (m, 4 H, Ar), 7.45 (s, 2 H, Ar), 7.18 (d, 1 H, J = 15.2, HC=), 3.52-3.42 (m, 4 H, 2 x $\mathrm{CH}_{2}$ ), 2.81-2.70 (m, $4 \mathrm{H}, 2$ x CH 2$), 1.99-1.86$ (m, $4 \mathrm{H}, 2 \times \mathrm{CH}_{2}$ ). 
(E)-2,4-bis(4-bromophenyl)-6-(2-(1,2,3,5,6,7-hexahydropyrido[3,2,1-ij]quinolin-9yl)vinyl)pyrylium boron tetrafluoride salt 6jc69-5

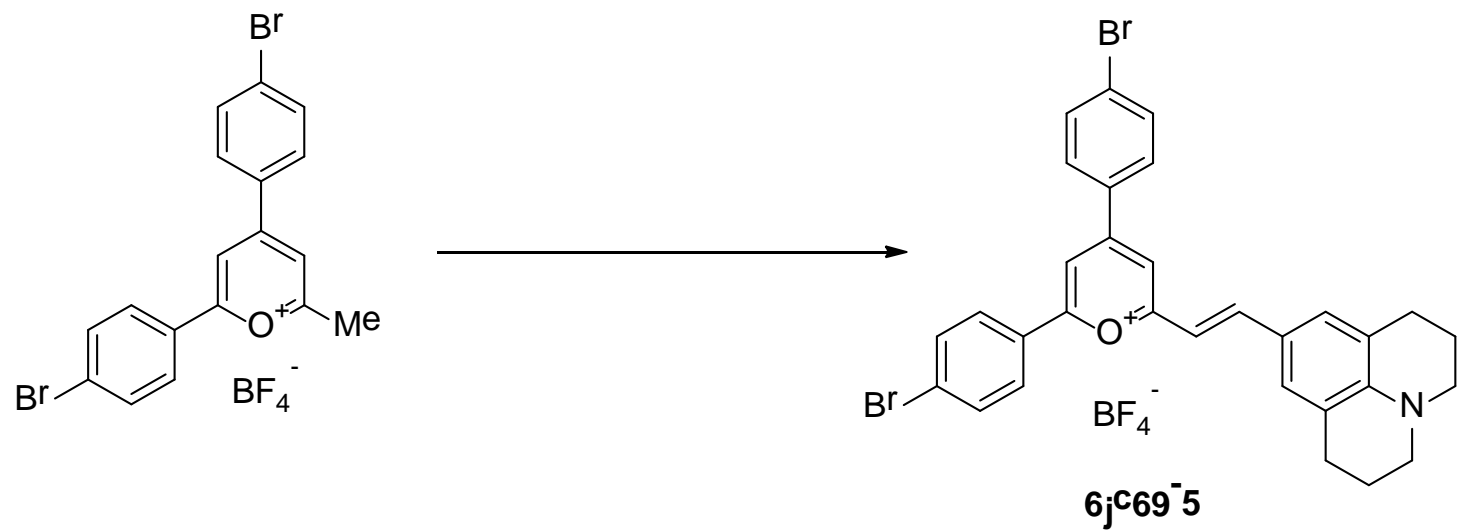

To pyrylium salt 6jc47 (59 mg, $0.12 \mathrm{mmol}$ ) and 9-CHO-julolidine (25 mg, $0.12 \mathrm{mmol}$ ) in $\mathrm{MeOH}$ $(8 \mathrm{~mL})$ was stirred at room temperature overnight. The solvent was removed and the residue was suspended in ether and filtered to give solid. Recrystallization from EtOH gave green solid of 47 mg.

$\delta_{\mathrm{H}}\left(\mathrm{DMSO}-\mathrm{d}_{6}, 400 \mathrm{MHz}\right)$ 8.36-8.13 (m, $\left.7 \mathrm{H}, \mathrm{Ar}, \mathrm{HC}=\right)$, 7.94-7.85 (m, $\left.4 \mathrm{H}, \mathrm{Ar}\right), 7.45$ (s, $2 \mathrm{H}, \mathrm{Ar}$ ), 7.18 (d, $1 \mathrm{H}, \mathrm{J}=14.8$, HC=), 3.52-3.43 (m, 4 H, 2 x CH ), 2.82-2.71 (m, 4 H, 2 x CH 2 ), 1.98-1.88 (m, $4 \mathrm{H}, 2 \times \mathrm{CH}_{2}$ ). 